\title{
Generalized Approach to Analysis of Multifractal Properties from Short Time Series
}

\author{
Lyudmyla Kirichenko ${ }^{1}$ \\ Department of Applied Mathematics \\ Kharkiv National University of \\ Radio Electronics \\ Kharkiv, Ukraine
}

\author{
Abed Saif Ahmed Alghawli ${ }^{2}$ \\ Department of Computer Science \\ College of Sciences and Humanities \\ Prince Sattam Bin Abdulaziz \\ University Aflaj \\ Kingdom of Saudi Arabia
}

\author{
Tamara Radivilova ${ }^{3}$ \\ V.V. Popovskyy Department of \\ Infocommunication Engineering \\ Kharkiv National University of \\ Radio Electronics \\ Kharkiv, Ukraine
}

\begin{abstract}
The paper considers a generalized approach to the time series multifractal analysis. The focus of research is on the correct estimation of multifractal characteristics from short time series. Based on numerical modeling and estimating, the main disadvantages and advantages of the sample fractal characteristics obtained by three methods: the multifractal fluctuation detrended analysis, wavelet transform modulus maxima and multifractal analysis using discrete wavelet transform are studied. The generalized Hurst exponent was chosen as the basic characteristic for comparing the accuracy of the methods. A test statistic for determining the monofractal properties of a time series using the multifractal fluctuation detrended analysis is proposed. A generalized approach to estimating the multifractal characteristics of short time series is developed and practical recommendations for its implementation are proposed. A significant part of the study is devoted to practical applications of fractal analysis. The proposed approach is illustrated by the examples of multifractal analysis of various real fractal time series.
\end{abstract}

Keywords-Fractal time series; multifractal analysis; estimation of multifractal characteristics; generalized Hurst exponent; practical applications of fractal analysis

\section{INTRODUCTION}

In the last years, there has been a growing interest in complex systems that have a fractal structure: informational, biological, physical, technological, financial and other. The dynamics of such systems generate time series with fractal (self-similar) properties. Time series fractal analysis is used to simulate, analyze and control complex systems in various fields [1]-[5]. Processes with fractal properties can be divided into two groups: monofractal and multifractal. Monofractal processes are homogeneous in the sense of fractal properties and have single scaling exponent. Multifractal processes have heterogeneous scale properties and are characterized by a set of scaling exponents.

There are a large number of methods for estimating the parameters of self-similar and multifractal processes from time series [6]-[11]. Methods based on wavelet transforms are of particular importance among the research methods of fractal nonstationary processes [2], [12], [13]. Currently, the two most popular tools of time series multifractal analysis are the method of multifractal detrended fluctuation analysis (MFDFA) and method of wavelet transform modulus maxima (WTMM). The MFDFA method is focused on time series with trend components and has been widely used due to [6], [14]. The WTMM method is based on continuous wavelet transform and was originally proposed in [15], [16]. Both methods are a powerful tool for the statistical processing of nonstationary processes. A significantly smaller number of studies are carried out using the method of multifractal analysis based on discrete wavelet transform [2], [17], [18].

In recent years, many studies were focused on the statistical properties of fractal characteristics estimates obtained by the above methods. However, most of the studies done have certain limitations. The main attention was paid to the statistics of estimates of self-similarity degree obtained by different methods [7], [8], [12], [18]. When studying the properties of multifractal characteristics, time series of great length were usually considered. So, for example, in [14], [19], when conducting a comparative analysis between MFDFA and WTMM, the length of the realizations was over 60 thousand values. In this case, the sample fractal characteristics are quite close to the theoretical ones. At the same time, the time series obtained in practice have a much smaller range of values. In [9], [20], aimed at studying the accuracy of estimating short time series, the minimum series length was over 1000 values, which is also quite large.

Many studies also compare the estimates of multifractal characteristics calculated using the same method [9], [14], [19]. The most common in practice is the MFDFA method. Quite a few researchers use several methods to evaluate the multifractal properties of time series. Despite numerous publications related to the practical application of fractal analysis, still, there is no universal approach to the estimation of fractal characteristics by time series. Besides, some practical questions of estimation from short non-stationary time series remain unsolved.

This work aims to study the application of multifractal analysis methods to short time series and to propose a generalized approach to the analysis of the multifractal properties of time series.

\footnotetext{
*Corresponding Author

Submission Date: April 20, 2020

Acceptance Date: May 12, 2020
} 


\section{SELF-Similarity PROPERTY AND ITS ESTIMATION METHODS}

\section{A. Definitions and Properties of Self-Similar Stochastic Processes}

A process $X(t)$ is called self-similar if it is invariant in distribution when changing the time-scale:

$$
\operatorname{Law}\{X(a t)\}=\operatorname{Law}\left\{a^{H} X(t)\right\}, \forall a>0, t>0
$$

The parameter $H$ is called Hurst exponent. It is a measure of self-similarity and together with that a measure of longrange dependence of process. In this case, the correlation function $K(\tau)$ of the process $X(t)$ decreases hyperbolically: $K(\tau) \sim \tau^{-\beta}$, where $H=1-(\beta / 2), 0<\beta<1$.

$X_{t}^{(m)}=\frac{1}{m}\left(X_{t m-m+1}+\ldots+X_{t m}\right), \quad m, t \in N$.

A process with discrete time $X$ is called self-similar with the parameter $H$ if the expression is true.

$\operatorname{Law}\left\{m^{1-H} X^{(m)}\right\}=\operatorname{Law}\{X\}$,

that is, the process does not change the distribution laws after averaging over blocks of length $m$.

The Hurst parameter $H$ is a measure of the long-term dependence duration. The case $0.5<H<1$ means that the process has persistent behavior or long memory. In other words, if positive (or negative) process increments have been observed for some time in the past, then with the probability close to the value $H$, this trend will continue.

The case $0<H<0.5$ means an antipersistent process. Here, high process values follow low ones, and vice versa. In other words, the probability that the process will change its trend in the opposite direction is as great as the parameter $H$ closer to 0 .

When $H=0.5$ the deviations of the process from the mean are indeed random and do not depend on the previous values, that corresponds to the case of the ordinary Brownian motion.

The $q$-moments of the self-similar random process $X(t)$ can be expressed as:

$\mathrm{E}\left[|X(t)|^{q}\right] \propto t^{q H}$

In particular, for the value $q=2$, we have a scaling relation for the variance.

$\operatorname{Var}[X(t)] \propto t^{2 H}$

Most methods for estimating the Hurst exponent are based on scaling change of some series characteristics close to variance. For example, in the method of R/S analysis change of the normalized range of the cumulative series $\frac{R}{S}(\tau) \propto \tau^{H}$ is investigated, in the method of detrended fluctuation analysis the fluctuation function $F(\tau) \propto \tau^{H}$ is investigated, etc.

The property of self-similarity corresponds to the linear dependence of the logarithm of the considered value, on the $\operatorname{logarithm}$ of the time $\log \tau$. For example, in cases of detrended fluctuation analysis, we have linear regression $\log F(\tau)=H \log (\tau)+$ const which is constructed by the least squares method. The self-similarity parameter $H$ can be estimated by the tangent of the line inclination angle.

\section{B. Methods for Estimating the Hurst Exponent}

To estimate the Hurst exponent of a time series, many methods have been proposed in [2], [6]-[8]. Consider the most popular.

Rescaled range method. This method was proposed by $\mathrm{H}$. Hurst and is currently the most well-known and popular method of fractal analysis [7], [8]. It is widely used in telecommunication technologies, in the study of the selfsimilar properties of information traffic, the study of the dynamics of financial markets, the study of biomedical signals, etc.

In this method in the study of a time series $x(t)$ of length $\tau$, the following relation is determined.

$$
\frac{R(\tau)}{S(\tau)}=R / S=\frac{\max \left(x^{\text {cum }}(t, \tau)\right)-\min \left(x^{c u m}(t, \tau)\right)}{\sqrt{\frac{1}{\tau-1} \sum_{t=1}^{\tau}(x(t)-\bar{x})^{2}}}, t=\overline{1, \tau},
$$

where $R(\tau)$ is a range of the cumulative series $x^{\text {cum }}(t, \tau)$; $S(\tau)$ is the standard deviation of the original series;

$\bar{x}(\tau)=\frac{1}{\tau} \sum_{t=1}^{\tau} x(t) ; x^{c u m}(t, \tau)=\sum_{i=1}^{t} x(i)-\bar{x}(\tau)$

For a self-similar process, this ratio for large values $\tau$ has the following scaling:

$\mathrm{E}\left[\frac{R}{S}\right] \propto \tau^{H}$

The dependence of $\frac{R(\tau)}{S(\tau)}$ on $\tau$ in double logarithmic scale for a self-similar time series, have to be a straight line, approximated by the least squares method. The value $H$ is calculated as the tangent of line angle dependencies $\log \frac{R(\tau)}{S(\tau)}$ on $\log (\tau)$, as it is shown in Fig. 1 .

The method of detrended fluctuation analysis (DFA). This method was originally proposed for the analysis of long-term correlations in the structure of the heart rhythm. Currently, it is one of the most widely used methods for studying various non-stationary time series [6], [14], [19]-[23]. 


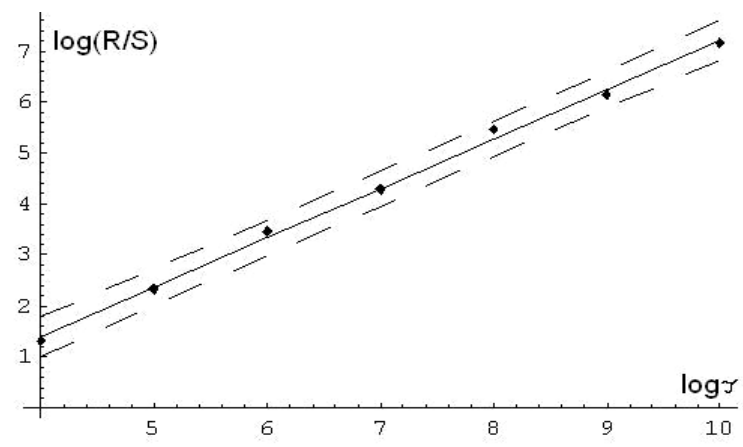

Fig. 1. The Dependence of $\log (R / S)$ on $\log (\tau)$, Obtained by Rescaled Range Method.

In the DFA method, for the initial time series $x(t)$, the cumulative series is constructed $y(t)=\sum_{i=1}^{t} x(i)$, which is divided into $N$ segments with a length $\tau$, and for each segment $y(t)$, the fluctuation function is calculated:

$F^{2}(\tau)=\frac{1}{\tau} \sum_{t=1}^{\tau}\left(y(t)-Y_{m}(t)\right)^{2}$,

where $Y_{m}(t)$ is a local $m$-polynomial trend that limited by this segment.

The function $F(\tau)$ averaged throughout the entire series $y(t)$ has a scaling dependence on the length of a series segment:

$F(\tau) \propto \tau^{H}$

The plot of dependence $\log F(\tau)$ on $\log \tau$ in a certain range of values is straight line approximated by the least squares method. The value $H$ is calculated as the tangent of straight line angle of dependence $\log F(\tau)$ on $\log \tau$, as it is shown in Fig. 2.

Discrete Wavelet Transform Method. The main development of the method was in the papers [2], [12]. Currently, it is used in the analysis of both stationary and nonstationary time series in various fields of research.

The method of wavelet estimation of self-similarity degree $H$ is based on the properties of the detail wavelet coefficients of time series decomposition by Discrete Wavelet Transform. The basis of this method is a statement that the wavelet energy value $E_{j}$ at the wavelet decomposition level $j$ satisfies the scaling relation:

$E_{j} \propto 2^{(2 H+1) j}$

The plot of dependence $\log _{2}\left(E_{j}\right)$ on $j$ is a straight line approximated by the least squares method. The value of exponent $H$ can be found by evaluating tangent of straight line angle. The wavelet energy spectrum of a self-similar process and dependence $\log _{2}\left(E_{j}\right)$ on $j$ are shown in Fig. 3 .

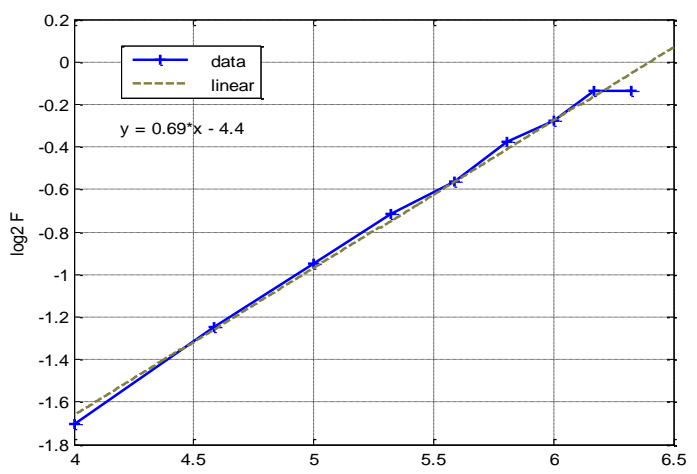

Fig. 2. Dependence $\log F(\tau)$ on $\log (\tau)$, Obtained by the DFA Method.

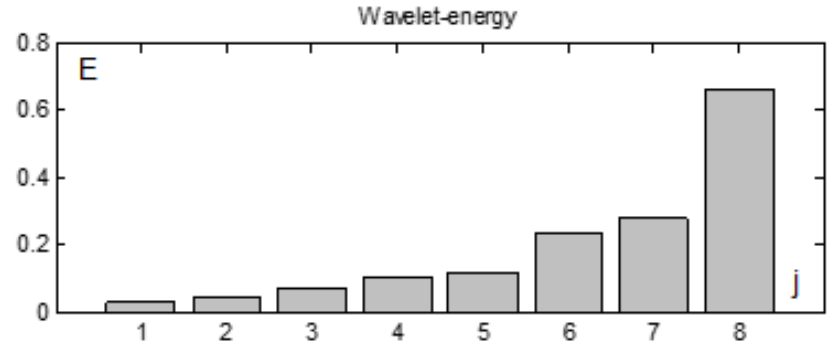

(a)

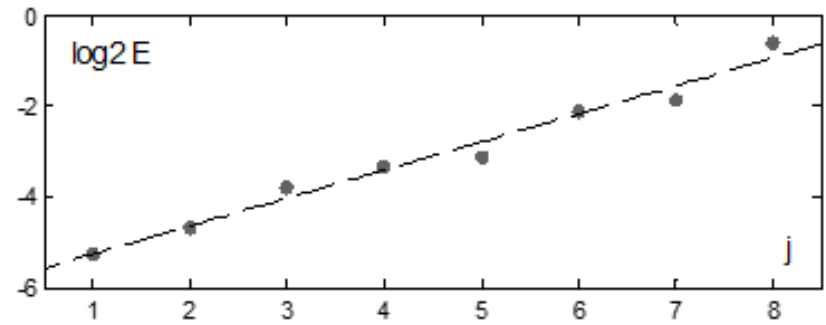

(b)

Fig. 3. The (a) Wavelet Energy Spectrum of a Self-Similar Process; (b) Dependence $\log _{2}\left(E_{j}\right)$.

\section{EStimation Methods OF MUlTifRactal CHARACTERISTICS}

\section{A. Definitions and Properties of Multifractal Stochastic Processes}

Multifractal stochastic processes also are invariant in distribution, but in this case, the change of quantitative characteristics of the process depends on the magnitude of the stretching in time.

$\operatorname{Law}\{X(a t)\}=\operatorname{Law}\{\mathrm{M}(a) \cdot X(t)\}, \forall a>0, t>0$

where $\mathrm{M}(a)$ is a random function which independent of $X(t)$. In the case of a self-similar (monofractal) process $\mathrm{M}(a)=a^{H}$.

For multifractal processes, the following relationship of $q$ moments holds:

$\mathrm{E}\left[|X(t)|^{q}\right] \propto t^{q\lceil h(q)}$, 
where $h(q)$ is a function of generalized Hurst exponent. Value $h(q)$ at $q=2$ is equal to the degree of self-similarity $H$. Generalized Hurst exponent of the monofractal process does not depend on the parameter $q: h(q)=H$. For the case $q=2$, it again gets at the formula (5).

Thus, before investigating the multifractal properties of the time series, it is necessary to determine the existence of selfsimilar properties. A more detailed analysis of the selfsimilarity of time series is presented in [21]. Consider some popular methods of time series estimation of multifractal properties.

\section{B. The Method of Multifractal Detrended Fluctuation Analysis}

When performing multifractal fluctuation detrended analysis (MFDFA), the dependence of the fluctuation function $F_{q}(s)$ on the parameter $q$ is investigated:

$F_{q}(s)=\left\{\frac{1}{N} \sum_{i=1}^{N}\left[F^{2}(s)\right]^{\frac{q}{2}}\right\}^{\frac{1}{q}}$

$F_{q}(s)$ is obtained by raising the expression (7) to the power $q$ and then averaging over all segments. Since at $q=0$ (12) contains uncertainty, the following expression is usually used instead:

$F_{q}(\tau)=\operatorname{Exp}\left\{\frac{1}{N} \sum_{i=1}^{N} \ln \left[F^{2}(\tau)\right]\right\}$

Changing the length of the segment $s$ at a fixed $q$, we find the dependence $F_{q}(s)$. If the time series has multifractal properties, then the fluctuation function is represented by a power dependence:

$F_{q}(s) \propto s^{h(q)}$,

where $h(q)$ is a function of generalized Hurst exponent.

For the monofractal time series, the fluctuation function $F_{q}(s)$ is the same for all segments and the generalized Hurst exponent does not depend on the parameter $q: h(q)=H$. For multifractal series, $h(q)$ is a non-linear function: with positive $q$, the main contribution to the function $F_{q}(s)$ is provided by segments that exhibit large deviations $F^{2}(s)$, while for negative $q$ the segments with small $F^{2}(s)$ dominate.

Fig. 4 shows the fluctuation functions $F_{q}(s)$ for the monofractal (a) and multifractal (b) processes of the parameter values $q=\{-5,-2,0,2,5\}$.

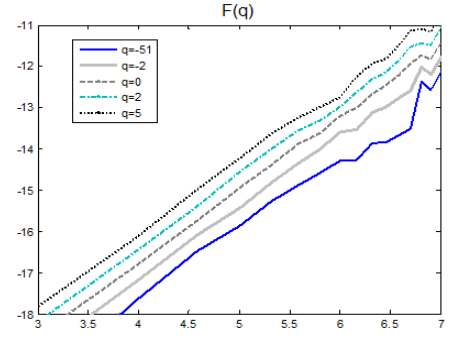

(a)

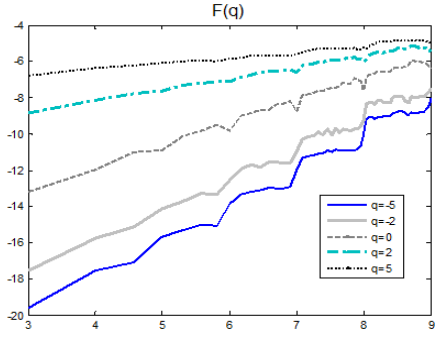

(b)
Fig. 4. Functions $F_{q}(s)$ for (a) the Monofractal and (b) Multifractal Time Realizations.

We can offer the following step-by-step algorithm for the estimation of the multifractal characteristics of time series $X(t), \quad t=1,2, \ldots, n$.

1. For the calculation, it is necessary to convert the original series to cumulative $X^{\text {cum }}(k)=\sum_{t=1}^{k} X(t), k=1,2, \ldots, n$. If the original time series is cumulative, this step is skipped.

2. The range $q$ is specified, in which it is required to determine the generalized Hurst exponent $h(q)$.

The series $X^{\text {cum }}(t)$ is divided into $N$ non-overlapping segments of length $\tau$ and fluctuation function is calculated for each segment where $Y m(t)$ is a local $m$-polynomial trend within a given segment.

3. The function $F(\tau)$ is averaged over the whole series $X^{\text {cum }}(t)$ :

$F(\tau)=\sqrt{\frac{1}{N} \sum_{i=1}^{N} F_{i}^{2}(\tau)}$

4. The fluctuation function is calculated for value $q$ :

$F^{2}(\tau)=\frac{1}{\tau} \sum_{t=1}^{\tau}\left(X^{c u m}(t)-Y_{m}(t)\right)^{2}$

5. The value $\tau$ increases and steps $3-5$ are repeated.

6. Linear regression $\log F_{q}(\tau)=k \log (\tau)+b$ is constructed by the least squares method. The value $k$ is equal to the value of $h(q)$ for a given value $q$.

7. Performing steps 2-6 for all given values of the parameter $q$, the function $h(q)$ is obtained.

\section{Wavelet Transform Modulus Maxima Method}

The method of wavelet transform modulus maxima (WTMM) is based on the mathematical apparatus of wavelet analysis. The continuous wavelet transform of the function $X(t)$ is described by 
$W(a, b)=\frac{1}{\sqrt{a}} \int_{-\infty}^{\infty} X(t) \psi_{a b}(t) d t$

where $W(a, b)$ is the wavelet spectrum, $\psi_{a b}(t)$ is the wavelet function with scale $a$ and shift $b$.

The WTMM algorithm involves the study of the behavior of a function $X(t)$ in two stages. At the first stage, the wavelet transform (14) is performed. The result of the wavelet transform is the wavelet spectrum $W(a, b) . W(a, b)$ can be represented as the surface of the wavelet coefficients in threedimensional space. An example of multifractal time series is shown in Fig. 5(a). The surface of wavelet coefficients for this time series is shown in Fig. 5(b). The most important information is contained in local extremum lines of function $W(a, b)$ (it is shown in Fig. 5(c)), which are searched at every scale $a$

The choice of a suitable wavelet function is determined by several aspects. This function must have $m$ zero moments [2]. On the one hand, large $m$ allows ignoring large-scale polynomial trends and analyzing small-scale variations of the function $X(t)$. On the other hand, an increase in the number of zero moments leads to an increase in the number of local extrema lines and the appearance of a large number of additional lines terminating on small scales [24]. Such lines are too short for estimating power dependences and become hindrances in the numerical analysis of singularities.

Usually, wavelets of the Gauss family are used as wavelet functions [15], [19], [24]. If high order derivatives of the Gauss function are taken, then the number of additional short lines of local maxima increases, which is due to oscillating "tails" of wavelet functions. Therefore, when conducting a multifractal analysis, it is advisable to limit the value $m=2$.

The basic information about possible local features of $X(t)$ at a point $t_{0}$ lies in the asymptotic behavior of the coefficients $W\left(a, t_{0}\right)$ at small scales $a$. If the coefficients on a small scale diverge, $X(t)$ has a feature in $t_{0}$. If the coefficients $W\left(a, t_{0}\right)$ are close to zero in a neighborhood $t_{0}$ on a small scale, then $X(t)$ is regular at this point. The first stage of the WTMM algorithm is completed by construction of the "skeleton" of the coefficients $W(a, b)$.

The second stage of the WTMM algorithm is to calculate the partition function:

$$
Z(q, a)=\sum_{l \in L(a)}\left(\sup _{a^{\prime} \leq a}\left|W\left(a^{\prime}, x_{l}\left(a^{\prime}\right)\right)\right|\right)^{q},
$$

where $L(a)$ is the set of all lines maxima $l$ of the wavelet coefficients modules of the scale $a ; x_{l}(a)$ is the location of the maximum at this scale.

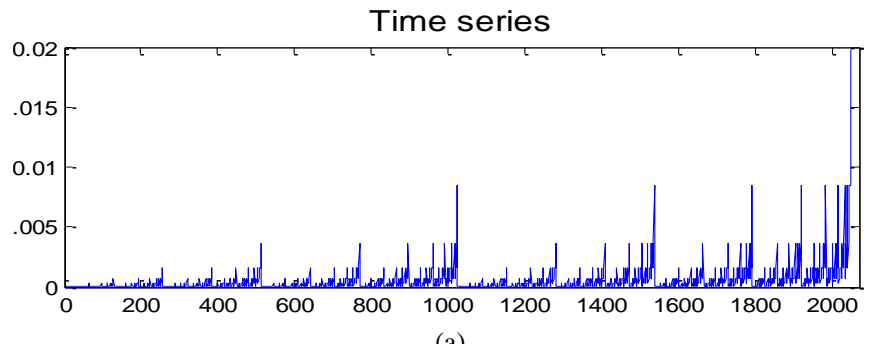

(a)

By scale Values of $\mathrm{Ca}, \mathrm{b}$ Coefficients for $\mathrm{a}=13579 \ldots$

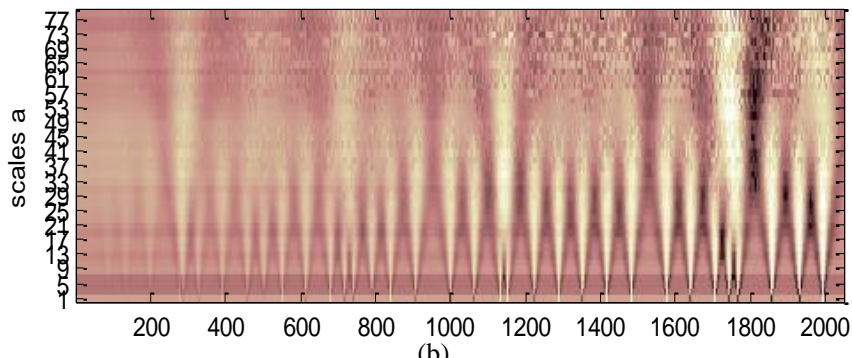

(b)

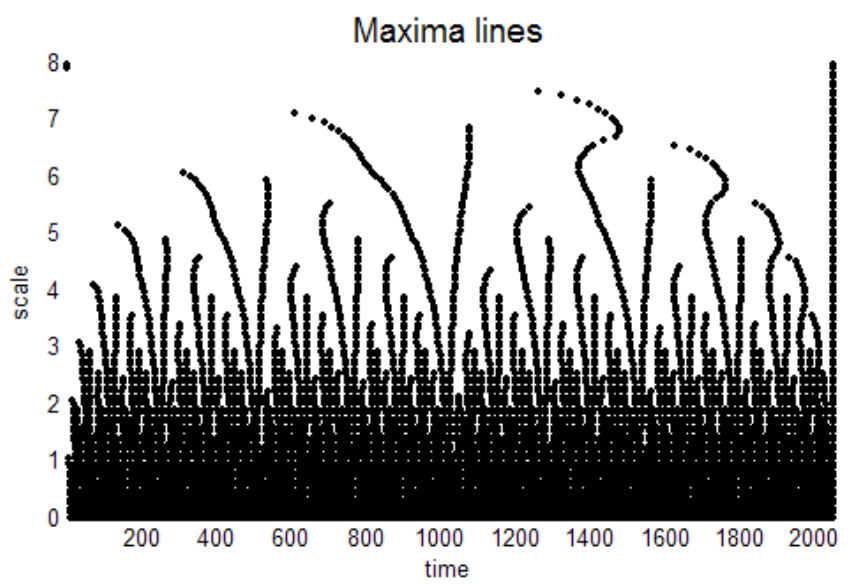

(c)

Fig. 5. (a) The Time Series, (b) The Surface of the Wavelet Coefficients, (c) The Lines of Local Maxima.

To calculate $Z(q, a)$ the maximum value of the wavelet coefficients modulus along each line on scales smaller than the specified scale $a$ is chosen. In this case, the following relation holds:

$Z(q, a) \propto a^{\tau(q)}$,

where $\tau(q)$ is scaling exponent that is related to the generalized Hurst exponent $h(q)$ by the ratio [14]:

$\tau(q)=q h(q)-1$,

The multifractal analysis based on the wavelet transform allows studying singularities with negative values of $q$ [15,16]. Partial functions $Z(q, a)$ at $q<0$ characterizing scaling features for weak singularities (small fluctuations), and at $q>0$ for strong singularities (large fluctuations). 
The statistical sums $Z(q, a)$ of a realization of the multifractal process for the values $q=\{-5,-2,0,2,5\}$ are shown in Fig. 6(a). The scaling exponents $\tau(q)$ of multifractal and monofractal realizations are shown in Fig. 6(b).

The next step-by-step algorithm can be offered to estimate the multifractal characteristics of time series $X(t), \quad t=1,2, \ldots, n$ by WTMM.

1. It is necessary to convert the original series to cumulative $X^{c u m}(k)=\sum_{t=1}^{k} X(t), k=1,2, \ldots, n$ for the calculation. If the original time series is cumulative, this step is skipped.

2. The wavelet transform by (14) is performed and the spectrum of wavelet coefficients $W(a, b)$ is found for this series.

3. A set of local maximum lines is determined for the surface $W(a, b)$.

4. The range $q$ is specified, where it is required to determine the scaling exponent $\tau(q)$ and generalized Hurst exponent $h(q)$.

5. The partial function $Z(q, a)$ is calculated by (15) for given $q$ and $a$.

6. The linear regression $\log Z(q, a)=k \log (a)+b \quad$ is constructed by the least squares method. The value $k$ is equal the value of $\tau(q)$ for a given value $q$.

7. By performing steps 5-6 for all given values of the parameter $q$, we obtain the function $\tau(q)$.

8. The values of the generalized Hurst exponent $h(q)$ are determined by (17).

\section{Multifractal Analysis based on Discrete Wavelet Transform}

In subsection 2.2, a method of determining the degree of self-similarity using DWT is considered. It is based on the properties of the detail coefficients obtained by the time series decomposition.

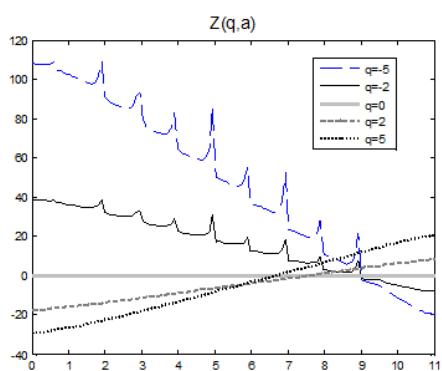

(a)

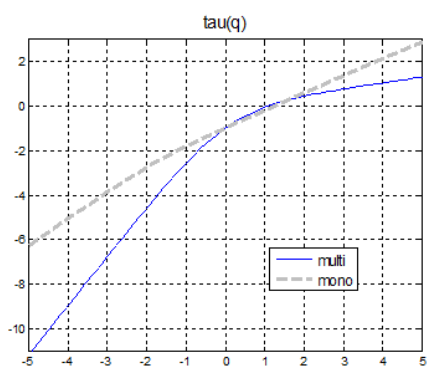

(b)
Fig. 6. (a) The Functions $Z(q, a)$ of Multifractal Realization with different $q$; (b) The Functions $\tau(q)$ of Multifractal and Monofractal Realizations.
Similarly, in $[17,18]$, a method of estimating multifractal characteristics based on DWT was proposed (MFDWT).

If for a multifractal process $X(t)$ there are $q$-th moments, then for the detail wavelet coefficients obtained by DWT, the following relation holds:

$\mathrm{E}|\operatorname{det}(j, k)|^{q}=\mathrm{E}|\operatorname{det}(0, k)|^{q} 2^{-j\left(\xi(q)-\frac{q}{2}\right)}$,

where $\operatorname{det}(j, k)$ is $k$-th detail wavelet coefficient of level $j, \xi(q)$ is a nonlinear function of a parameter $q$ associated with the generalized Hurst exponent $h(q)$ considering (17) by the expression:

$h(q)=\frac{\xi(q)+1}{q}-\frac{1}{2}$.

Based on the expressions (18-19) and taking into account the properties of the detail wavelet coefficients, the following step-by-step algorithm can be offered to estimate the multifractal characteristics of time series $X(t), \quad t=1,2, \ldots, n$ by MFDWT.

1. The original series has to be converted to cumulative series $X^{\text {cum }}(k)=\sum_{t=1}^{k} X(t), k=1,2, \ldots, n$. If the original time series is cumulative, this step is skipped.

2. The range $q$ is specified, in which it is required to determine the multifractal characteristics $\xi(q)$ and $h(q)$.

3. The range of decomposition levels of DWT is selected. The following value is calculated for each decomposition level $j$ :

$E_{j}^{(q)}=\frac{1}{N_{j}} \sum_{k=1}^{N_{j}}|\operatorname{det}(j, k)|^{q}$

4. Each value $j$ is assigned a logarithm of $E_{j}^{(q)}$. The tangent of the inclination angle of the approximating straight line is equal to value $\xi(q)$.

5. By performing steps 3-4 for all given values of the parameter $q$, the function $\xi(q)$ is obtained.

6. By (19) the corresponding values of the generalized Hurst exponent $h(q)$ are calculated.

The functions $\log _{2} E_{j}^{(q)}$ of the multifractal process realizations for the values $q=\{-5,-2,0,2,5\}$ are presented in Fig. 7(a). The functions $\xi(q)$ of different multifractal realizations are shown in Fig. 7(b). 


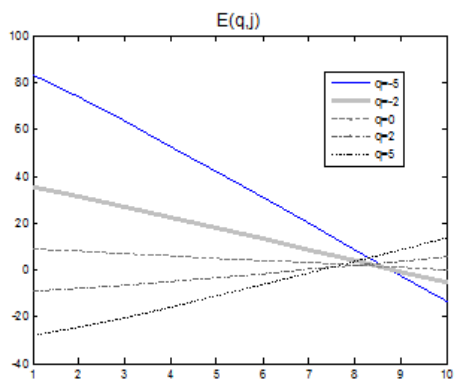

(a)

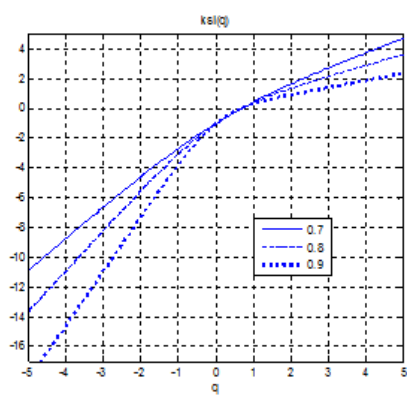

(b)
Fig. 7. (a) The Functions $\log _{2} E_{j}^{(q)}$ of Multifractal Realizations for $q=\{-5,-2,0,2,5\} ;$ b) the Functions $\xi(q)$ of Multifractal Realizations.

\section{Estimation of Multifractal Properties of Time SERIES}

\section{A. Experiment Description}

The section presents the results of a numerical experiment, during which the realizations of different fractal stochastic processes were modeled: fractal Brownian motion (monofractal process), $\alpha$-stable process (bi-fractal process) and binomial multiplicative stochastic cascade (multifractal process). The length of the realizations were chosen to be 250, 500, 1000 and 2000 values. For each generated time series, using the methods of MFDFA, WTMM and MFDWT the functions of the generalized Hurst exponent $h(q)$ were calculated. Then functions $h(q)$ were averaged over a set of realizations. The parameter $q$ had values in the range $-5 \leq q \leq 5$.

The following characteristic was chosen as a measure of proximity to theoretical values:

$$
\Delta h=\frac{1}{n_{q}} \sum_{q}\left|\hat{h}(q)-h_{T}(q)\right|,
$$

where $h_{T}(q)$ is the theoretical function of the generalized Hurst exponent; $\hat{h}(q)$ is sample function; $n_{q}$ is the number of values $q ; \Delta h$ is the average deviation of the sample value from the theoretical. Since the methods can have different errors for positive and negative values of parameter values $q$, it makes sense instead of $\Delta h$ to consider separately $\Delta h(q<0)$ and $\Delta h(q>0)$.

Model time series for estimation.

Fractal Brownian motion. FBM is the most famous and simple model of the self-similar process [25]. On the one hand, the FBM is a self-similar process with the Hurst exponent $H$. On the other hand, FBM can be considered as a monofractal process, in which the generalized Hurst exponent is a constant: $h(q)=H$.

Stable process with independent increments. A random process is a $\alpha$-stable process if its finite-dimensional distributions are stable random variables [26]. The parameter $\alpha$ is called the stability index and determines the heavy tails of the distribution. For a $\alpha$-stable process with independent increments, equality holds.

$$
\operatorname{Law}\{X(a t)\}=\operatorname{Law}\left\{a^{1 / \alpha} X(t)\right\}, \forall a>0, t>0
$$

It is shown that such processes are bi-fractal. The corresponding generalized Hurst exponent is described by:

$$
h(q)= \begin{cases}1 / \alpha & q \leq \alpha \\ 1 / q & q>\alpha\end{cases}
$$

Stochastic binomial cascade. The simplest model of a multifractal process is the deterministic binomial cascade [25]. When constructing it, the initial unit segment is divided into two equal intervals, weight coefficients $p_{1}$ and $p_{2}=1-p_{1}$ are assigned to each of them. As a result, in the second step, there are four intervals with weights $p_{1}^{2}, p_{1} p_{2}, p_{2} p_{1}$ and $p_{2}^{2}$. With an increase in the number of iterations, we obtain an ordered set of weights, which has multifractal properties. When constructing stochastic cascades, the weight coefficients are values of some random variable [27]. If a random variable with a beta distribution $\operatorname{Beta}(a, b)$ is used as a random variable, then in the case of $a=b$, the scaling exponent $\tau(q)$ can analytically determine over the interval of values $q>-1$ :

$$
\tau(\mathrm{q})=-\log _{2} \frac{\operatorname{Beta}(\alpha+\mathrm{q}, \alpha)}{\operatorname{Beta}(\alpha, \alpha)}-1
$$

The theoretical value of the function $h(q)$ is determined in accordance with (17).

\section{B. The Results of the Generalized Hurst Estimation Exponent by Time Series}

Estimation of multifractal characteristics for monofractal realizations.

A typical FBM realization with $\mathrm{H}=0.8$ is shown in Fig. 8(a) and the corresponding realization of increments (fractal Gaussian noise) is shown in Fig. 8(b). The length of realizations is 1000 values.

The sample values of the generalized Hurst exponent calculated by the methods MFDFA (a), WTMM (b) and MFDWT (c) are presented in Fig. 9. A straight line is the theoretical values $h(q)$. It is shown that with an increase in the length of the realization, sample characteristics tend to theoretical values. However, at small lengths, the obtained estimates demonstrate false multifractal properties. Therefore, in the case of the realization of a small length, it is necessary to conduct additional studies to confirm the monofractal properties.

Table I represents the values of the deviations $\Delta h$, calculated in accordance with (20). The estimates of the generalized Hurst exponent obtained by the MFDFA method are much closer to their theoretical values than the estimates calculated using wavelet transforms. Also, when using the WTMM method, determining the wavelet spectrum, local maxima lines and choosing the appropriate scale range is a 
more complicated task than determining the polynomial degree and the segment lengths in MFDFA. The MFDWT method demonstrates incorrect estimation of $h(q)$ with negative $q$, however, for positive $q$ the estimates by MFDWT method are close to the estimates by WTMM.

Estimation of multifractal characteristics for bi-fractal realizations.

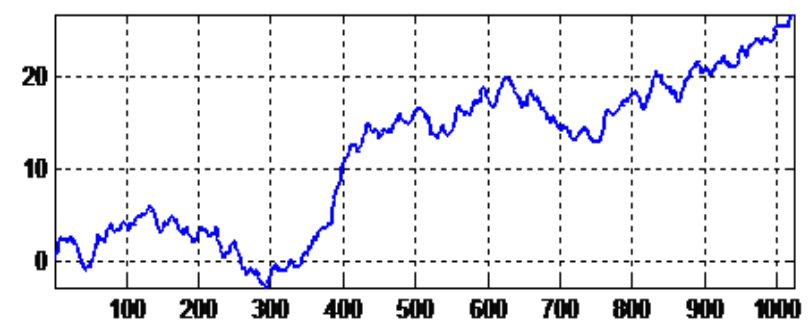

(a)

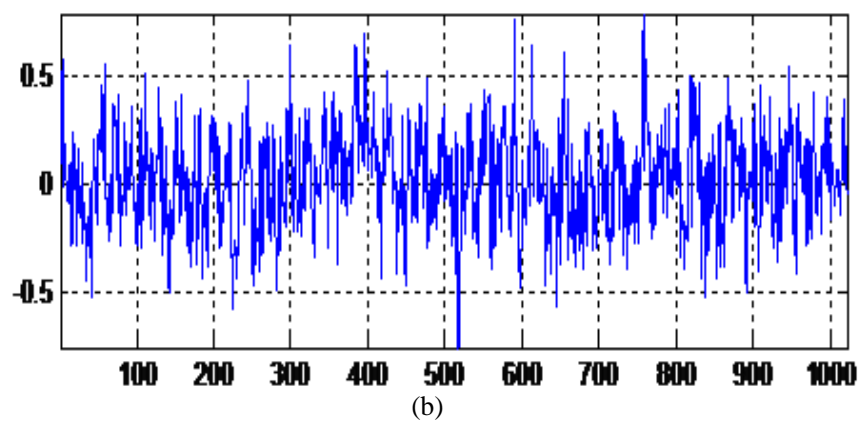

Fig. 8. (a) FBM Realization, (b) Realization of Increments.

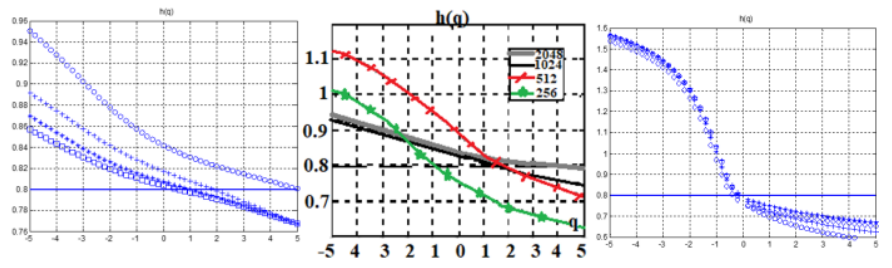

Fig. 9. Sample $h(q)$ of Monofractal Realization, Calculated by the Methods (a) MFDFA, (b) WTMM and (c) MFDWT.

TABLE I. DEVIATIONS OF SAMPLE $h(q)$ FOR MONOFRACTAL REALIZATIONS

\begin{tabular}{|l|l|l|l|}
\hline \multirow{3}{*}{ Length } & Method & $\Delta h(q<0)$ & $\Delta h(q>0)$ \\
\hline \multirow{3}{*}{250} & MFDFA & 0.094 & 0.018 \\
\cline { 2 - 4 } & WTMM & 0.18 & 0.13 \\
\cline { 2 - 4 } & MFDWT & 0.54 & 0.15 \\
\hline \multirow{3}{*}{500} & MFDFA & 0.06 & 0.015 \\
\cline { 2 - 4 } & WTMM & 0.3 & 0.06 \\
\cline { 2 - 4 } & MFDWT & 0.51 & 0.12 \\
\hline \multirow{3}{*}{1000} & MFDFA & 0.035 & 0.013 \\
\cline { 2 - 4 } & WTMM & 0.27 & 0.023 \\
\cline { 2 - 4 } & MFDWT & 0.50 & 0.1 \\
\hline \multirow{3}{*}{2000} & MFDFA & 0.025 & 0.014 \\
\cline { 2 - 4 } & WTMM & 0.15 & 0.018 \\
\cline { 2 - 4 } & MFDWT & 0.46 & 0.08 \\
\hline
\end{tabular}

Let us consider the estimation of $h(q)$ for the realizations of a $\alpha$-stable process. A typical realization of a $\alpha$-stable process with $\alpha=1.5$ is shown in Fig. 10(a) and the corresponding realization of increments is shown in Fig. 10(b). The length of realizations is 1000 values.

The sample functions of the generalized Hurst exponent obtained using the MPDFA, WTMM and MFDWT methods are shown in Fig. 11(a), (b) and (c), respectively.

The obtained estimates of $h(q)$ show false multifractal properties. The estimates obtained by the MFDFA method are closer to their theoretical values than the estimates calculated using wavelet transforms, as evidenced by Table II. The MFDWT method, as well as in the monofractal case, demonstrates incorrect estimation of multifractal properties. However, for positive $q$ the estimates of $h(q)$ by the MFDWT method have the smallest difference with the theoretical values.

Estimation of multifractal characteristics for realizations of a stochastic binomial cascade.

Let consider the estimation of multifractal properties for realizations of stochastic multiplicative binomial cascades whose weights have a beta distribution. For comparison with analytical characteristics (21), calculations were performed in the range $-1<q<5$.

The typical realization of a binomial stochastic cascade with a length of 1000 values is shown in Fig. 12(a). In this case the weights have a uniform distribution (i.e. $a=b=1$ ). The corresponding sample values of the generalized Hurst exponents are shown in Fig. 12(b) by MFDFA, (c) WTMM and (d) MFDWT. The theoretical function $h(q)$ is shown by the thin line.

The numerical values of the deviations are given in Table III. The WTMM method shows the worst estimation of the stochastic multifractal cascades. The MFDWT method shows good results and it is not inferior to the MFDFA when evaluating multifractal realizations.

Table IV shows the deviations values for the positive $q$ of all types of fractal time series. This is quite convenient and clear for a general results comparison. The estimation analysis showed that the estimates of the generalized Hurst exponent obtained from short time series have shifts that decrease with the increasing length of the time series. The method MFDFA has significant advantages in accuracy when estimating from short realizations, especially in the case of monofractal ones.
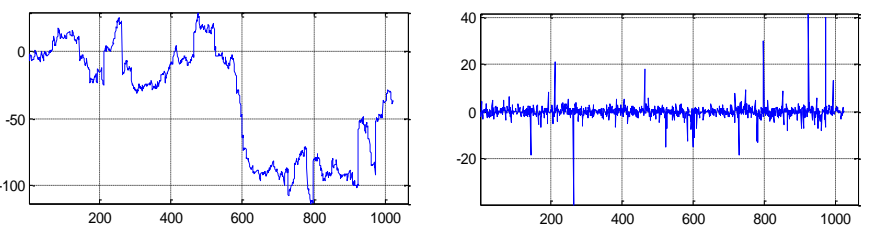

a) b)

Fig. 10. (a) Realization of a $\alpha$-Stable Process, (b) Realization of Increments. 


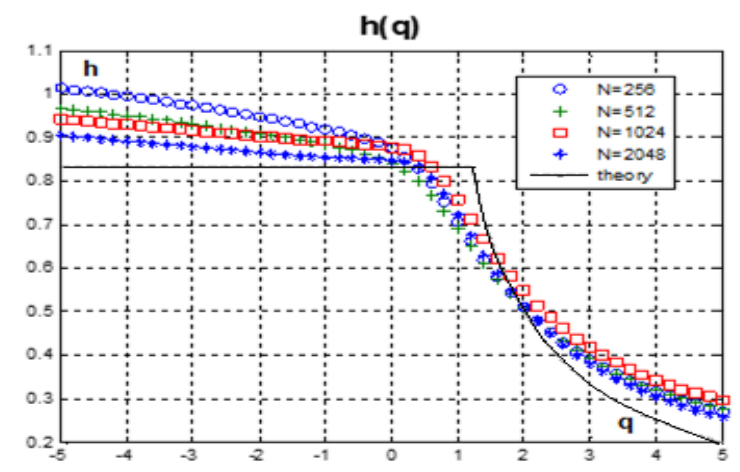

(a)

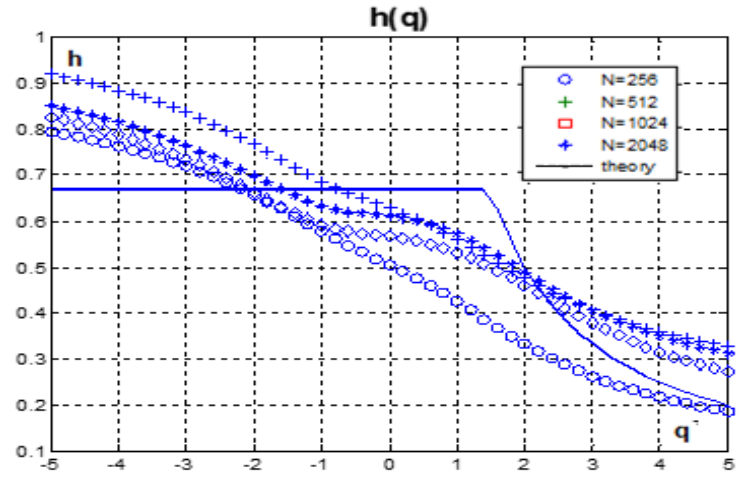

(b)

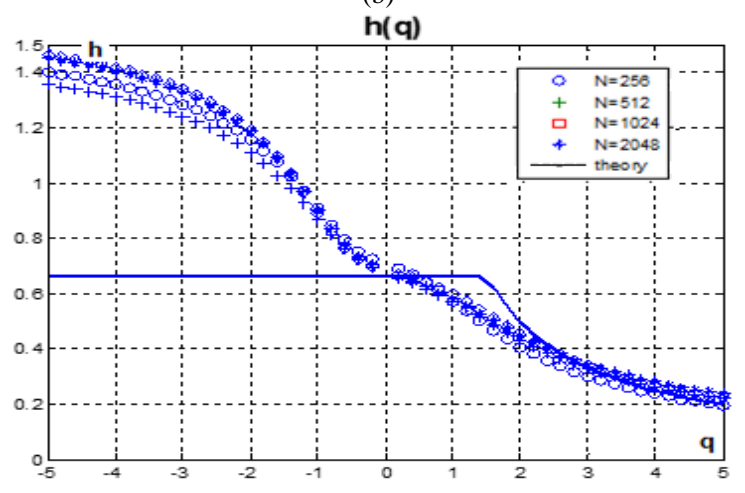

(c)

Fig. 11. Sample $h(q)$ of bi-fractal Realizations, Calculated by the Methods (a) MFDFA, (b) WTMM, (c) MFDWT.

TABLE II. DEVIATIONS OF SAMPLE $h(q)$ FOR BI-FRACTAL REALIZATIONS

\begin{tabular}{|l|l|l|l|}
\hline Length & Method & $\Delta h(q<0)$ & $\Delta h(q>0)$ \\
\hline \multirow{3}{*}{200} & MFDFA & 0.096 & 0.056 \\
\cline { 2 - 4 } & WTMM & 0.076 & 0.12 \\
\cline { 2 - 4 } & MFDWT & 0.47 & 0.047 \\
\hline \multirow{3}{*}{500} & MFDFA & 0.064 & 0.053 \\
\cline { 2 - 4 } & WTMM & 0.13 & 0.086 \\
\cline { 2 - 4 } & MFDWT & 0.45 & 0.042 \\
\hline \multirow{3}{*}{1000} & MFDFA & 0.057 & 0.05 \\
\cline { 2 - 4 } & WTMM & 0.08 & 0.075 \\
\cline { 2 - 4 } & MFDWT & 0.52 & 0.038 \\
\hline \multirow{3}{*}{2000} & MFDFA & 0.054 & 0.041 \\
\cline { 2 - 4 } & WTMM & 0.08 & 0.07 \\
\cline { 2 - 4 } & MFDWT & 0.54 & 0.034 \\
\hline
\end{tabular}

TABLE III. DEVIATIONS OF SAMPLE $h(q)$ FOR REALIZATIONS OF Stochastic Multifractal Cascade

\begin{tabular}{|l|l|l|l|}
\hline Length & Method & $\Delta h(q<0)$ & $\Delta h(q>0)$ \\
\hline \multirow{4}{*}{250} & MFDFA & 0.18 & 0.023 \\
\cline { 2 - 4 } & WTMM & 0.8 & 0.11 \\
\cline { 2 - 4 } & MFDWT & 0.17 & 0.016 \\
\hline \multirow{4}{*}{500} & MFDFA & 0.14 & 0.016 \\
\cline { 2 - 4 } & WTMM & 0.5 & 0.09 \\
\cline { 2 - 4 } & MFDWT & 0.17 & 0.016 \\
\hline \multirow{3}{*}{1000} & MFDFA & 0.16 & 0.012 \\
\cline { 2 - 4 } & WTMM & 0.56 & 0.077 \\
\cline { 2 - 4 } & MFDWT & 0.18 & 0.011 \\
\hline \multirow{3}{*}{2000} & MFDFA & 0.18 & 0.010 \\
\cline { 2 - 4 } & WTMM & 0.4 & 0.061 \\
\cline { 2 - 4 } & MFDWT & 0.15 & \\
\hline
\end{tabular}

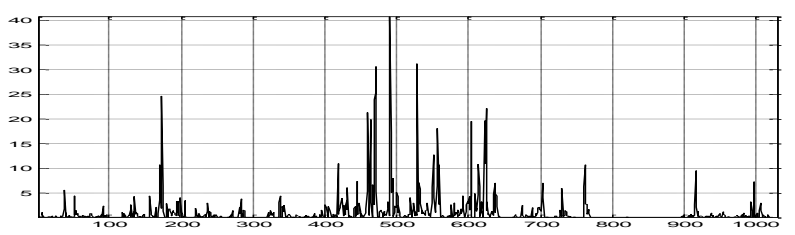

(a)

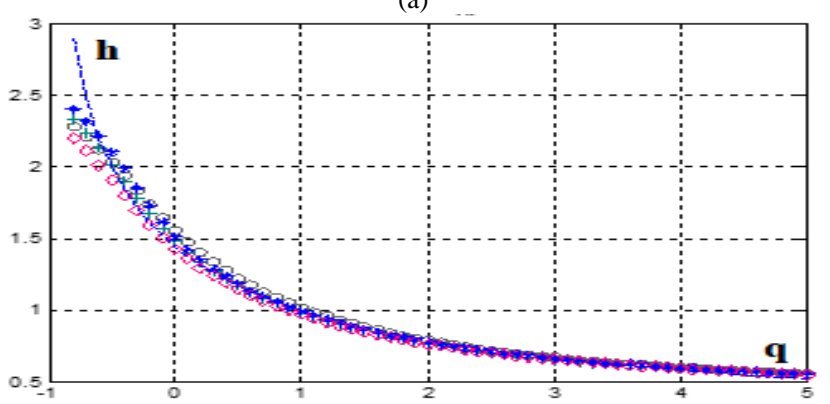

(b)

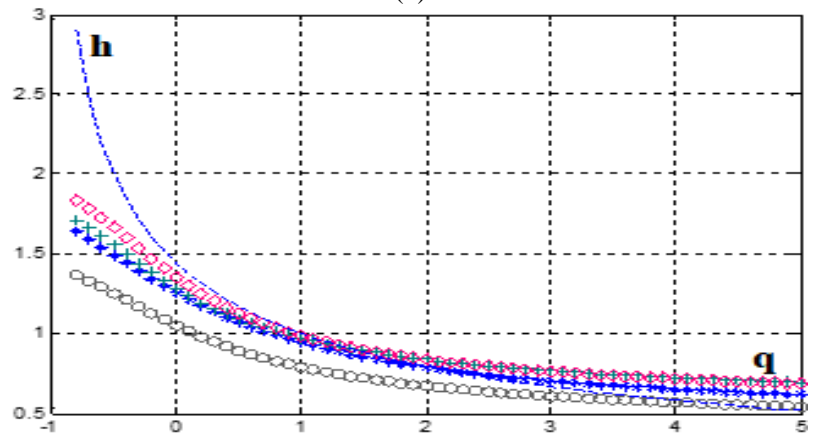

(c)

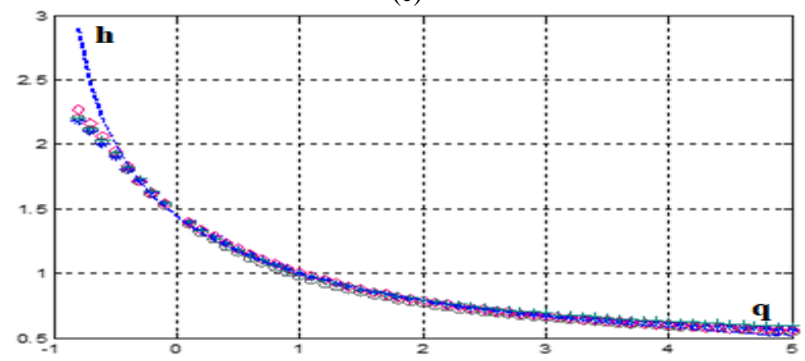

(d)

Fig. 12. (a) The Stochastic Cascade Realization; $h(q)$ Obtained by (b) MFDFA, (c) WTMM, (d) MFDWT. 
TABLE IV. DEVIATIONS $\Delta h(q>0)$ FOR MONOFRACTAL, BI-FRACTAL, AND MulTiFraCtal REALIZATIONS

\begin{tabular}{|l|l|l|l|l|}
\hline Length & Method & Monofractal & Bifractal & Multifractal \\
\hline \multirow{3}{*}{200} & MFDFA & 0.018 & 0.056 & 0.023 \\
\cline { 2 - 5 } & WTMM & 0.13 & 0.12 & 0.11 \\
\cline { 2 - 5 } & MFDWT & 0.15 & 0.047 & 0.024 \\
\hline \multirow{3}{*}{500} & MFDFA & 0.015 & 0.053 & 0.016 \\
\cline { 2 - 5 } & WTMM & 0.06 & 0.086 & 0.09 \\
\cline { 2 - 5 } & MFDWT & 0.12 & 0.042 & 0.016 \\
\hline \multirow{3}{*}{1000} & MFDFA & 0.013 & 0.05 & 0.012 \\
\cline { 2 - 5 } & WTMM & 0.023 & 0.075 & 0.077 \\
\cline { 2 - 5 } & MFDWT & 0.10 & 0.038 & 0.011 \\
\hline \multirow{3}{*}{2000} & MFDFA & 0.010 & 0.041 & 0.010 \\
\cline { 2 - 5 } & WTMM & 0.018 & 0.07 & 0.061 \\
\cline { 2 - 5 } & MFDWT & 0.08 & 0.034 & 0.01 \\
\hline
\end{tabular}

C. Testing the Hypothesis about Monofractality of Time Series

The results of the multifractal analysis of model time series demonstrate the need to develop a tool to distinguish between mono- and multifractal time series. Since the MFDFA method showed the best results in evaluating monofractal realizations, a method based on estimates obtained by MFDFA is proposed. It allows to accept or reject the hypothesis of the presence of time series monofractal properties.

For self-similar processes, the question of the estimates Hurst exponent $H$ distribution was considered in several publications [2], [8], [19], where it was shown, that estimates are normal random variables. The analysis of the sample distribution of the generalized Hurst exponent $h(q)$ showed that the estimates at $q>0$ have a normal distribution, the parameters of which depend on the value $q$. For $q<0$ the sample values $h(q)$ in the general case are not normal random variables.

The multifractal time series have a much greater difference in values $\Delta h=h(q 1)-h(q 2)$ than monofractal ones. A random variable $\Delta h$ has a normal distribution $N\left(m_{h}, s_{h}\right)$, the parameters of which depend on the length of the time series and the values $q$. The criterion of the monofractal degree was proposed to be considered the value $\Delta h=h(0.1)-h(5)$. By numerical simulation of monofractal processes with varying degrees of self-similarity, the sample values $m_{h}$ and $s_{h}$ were obtained. Table $\mathrm{V}$ presented these values for the series of different lengths $N$.

The same characteristics calculated from cascade processes with different degrees of heterogeneity, given by the beta distribution parameter $a$, are also presented in Table V.

The table shows that even with very weak multifractal properties of the process, mono- and multifractal time series can be distinguished. Thus, the value of a random variable $\Delta h$ can be used as test statistics for acceptance of the monofractal properties hypothesis. In this case, the null hypothesis is the assumption that the series is monofractal. Having obtained the estimate $\hat{h}(q)$ using the MFDFA method, the observable value $\Delta \hat{h}=\hat{h}(0.1)-\hat{h}(5)$ is calculated. The hypothesis is accepted with a significance level $\alpha$ if the observable value is within the range of acceptable values:

$$
\Delta \hat{h}<m_{h}(N)+t_{\alpha} s_{h}(N),
$$

where $N$ is the length of time series; $m_{h}$ and $s_{h}$ are values calculated for the monofractal process; $t_{\alpha}$ is the corresponding quantile of standard normal distribution.

TABLE V. PARAMETERS OF THE RANDOM VARIABLE $\Delta h$

\begin{tabular}{|c|c|c|c|c|c|c|c|c|}
\hline & \multicolumn{2}{|c|}{$\mathrm{N}=\mathbf{2 5 0}$} & \multicolumn{2}{|c|}{$\mathrm{N}=500$} & \multicolumn{2}{|c|}{$\mathrm{N}=1000$} & \multicolumn{2}{|c|}{$\mathrm{N}=2000$} \\
\hline & $m_{h}$ & $s_{h}$ & $m_{h}$ & $s_{h}$ & $m_{h}$ & $s_{h}$ & $m_{h}$ & $s_{h}$ \\
\hline $\begin{array}{l}\text { Monofracta } \\
1\end{array}$ & $\begin{array}{l}0.07 \\
5\end{array}$ & $\begin{array}{l}0.05 \\
2\end{array}$ & $\begin{array}{l}0.0 \\
5\end{array}$ & $\begin{array}{l}0.03 \\
8\end{array}$ & $\begin{array}{l}0.03 \\
5\end{array}$ & $\begin{array}{l}0.02 \\
6\end{array}$ & $\begin{array}{l}0.0 \\
3\end{array}$ & $\begin{array}{l}0.02 \\
2\end{array}$ \\
\hline \multicolumn{9}{|l|}{ Multifracta } \\
\hline$a=1$ & 0.87 & 0.23 & $\begin{array}{l}0.8 \\
6\end{array}$ & 0.18 & 0.85 & 0.16 & $\begin{array}{l}0.8 \\
2\end{array}$ & 0.12 \\
\hline$a=5$ & 0.32 & 0.13 & $\begin{array}{l}0.2 \\
9\end{array}$ & $\begin{array}{l}0.09 \\
5\end{array}$ & 0.28 & 0.08 & $\begin{array}{l}0.2 \\
7\end{array}$ & 0.07 \\
\hline$a=10$ & 0.23 & 0.1 & 0.2 & 0.07 & 0.18 & 0.06 & $\begin{array}{l}0.1 \\
7 \\
\end{array}$ & 0.05 \\
\hline
\end{tabular}

D. A Generalized Approach to Estimating the Multifractal Properties of Small Length Time Series

Summing up the research results, the following scheme for multifractal analysis of some random process represented by a time series $X(t)$ of length $N$ can be proposed. It should be noted that before proceeding to fractal analysis, it is necessary to find out from a priori known information whether the series is cumulative (for example, the exchange rate) or increments (for example, information traffic).

The main stages of fractal analysis can involve various methods of estimation. Since the application of the wavelet transform apparatus requires appropriate software and experience, the description of the scheme is structured in such way that the use of wavelet estimation methods is a desirable but not necessary element. However, the application of the MFDFA method is necessary for two reasons: this method has sufficient accuracy and is designed for non-stationary time series. Consider the step by step implementation of the generalized approach to the estimation of the multifractal properties of self-similar time series.

1) A preliminary study of the time series structure is a necessary stage of multifractal analysis, its full description is given in [21] This stage includes determining intervals of various scaling, detecting and removing short-term autoregressive dependence, estimation of the Hurst exponent $H$.

2) All three considered methods MFDFA, WTMM, and MFDWT are intended for analysis non-stationary series. But before proceeding to an estimation of multifractal properties, it is necessary to investigate time series structure by correlation function, Fourier spectrum or spectrum of wavelet energy, which allow identifying the trend and cyclical 
components of the series. The results of the preliminary study allow selecting the input parameters correctly for the multifractal analysis method.

3) When estimating the generalized Hurst exponent using the MFDFA method, it is necessary to first carry out a rough estimation of the Hurst exponent $H=h(2)$ using local polynomials of increasing degree and determine the degree to estimation. After that, evaluate the function $h(q)$, removing the local polynomial trend of this degree.

4) When evaluating the scaling exponent $\tau(q)$ by the WTMM method, it needs to select the appropriate scale range $a_{1} \leq a \leq a_{2}$ on which the local extremums of the function $W(a, b)$ are determined. The right choice of the scale range allows ignoring the influence of the trend components of the time series. Then it is necessary to carry out a rough estimation of the partial function $Z(q, a)$ to make sure that there are intervals of linear dependence $\log Z(q, a)$ on $\log a$.

5) When estimating the scaling exponent $\xi(q)$ using the MFDWT method, it needs to first select the appropriate range of values of levels $j_{1} \leq j \leq j_{2}$ at which there is a linear dependence $\log _{2} E_{j}$ on the level number $j$.

6) With weakly expressed multifractal properties, it is necessary to test the hypothesis of the monofractal properties of time series.

7) If estimates of multifractal characteristics were obtained by different methods, then to increase accuracy, it is desirable to conduct comparative analysis taking into account a priori information and the results of numerical studies.

\section{Fractal ANAlysis of REAL TIME SERIES}

\section{A. Fractal Analysis of Temperature Series}

Well-known time series with the property of self-similarity are temperature series. The specialized site [28] presents daily temperature series (maximum, minimum and average values) for different cities over 50 years from 1942 to 1992. Fig. 13 shows a fragment of the temperature series for 1952-1956 (a) and the corresponding series of daily increments (b).
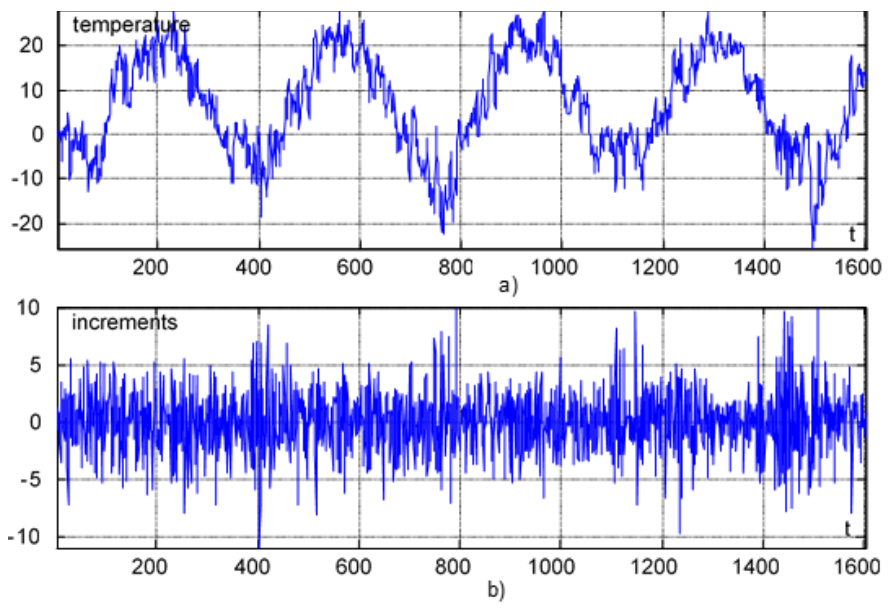

Fig. 13. A Series of Daily Temperatures (a) and a Series of Daily Increments (b).
Before the multifractal analysis, the fluctuation function $F(\tau)$ was investigated. The presence of a section with a linear dependence of $F(\tau)$ corresponds to the self-similar behavior of the time series. If the function $F(\tau)$ has several linear sections, this implies the several time scaling for different time intervals. Fig. 14(a) shows a graph $F(\tau)$ plotted over a time interval from $2^{5}$ (month) to $2^{9.5}$ (two years) days.

It is obvious that the graph has two sections close to linear with different tilt angles. It is worth noting the high expulsion in the second section when the value of the argument is equal to $2^{8.5}$ which is almost equal to one year and corresponds to the annual seasonal component of the time series. Multifractal analysis by the MFDFA method was carried out separately for each section. For the first section, the Hurst exponent $H=0.21$, for the second $H=0.92$. Fig. 14(b) shows the multifractal spectrum functions for both sections.

Thus, it can be concluded that at intervals from two months to six months (Section 1), the series of temperature dependence is antipersistent, and at time values from six months to one and a half years (Section 2), the series has a strong long-term dependence.

The research of the monofractal property, carried out by the method presented in subsection 4.3, has shown that in both cases the series has almost monofractal properties. Table VI shows the results of hypothesis testing for the generalized Hurst exponent sample values $\hat{h}(q)$ of average daily temperatures series. Critical values $\Delta h$ were obtained based on calculated data for the significance level $\alpha=0.05$ and the corresponding series length.

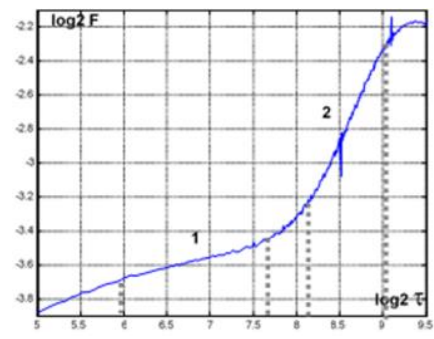

(a)

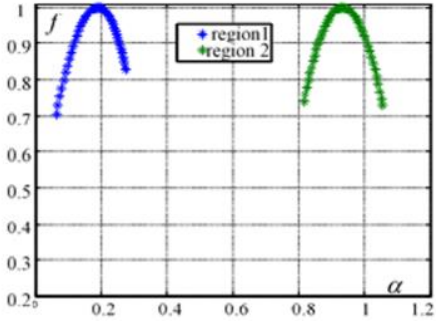

(b)
Fig. 14. The Fluctuation Function $F(\tau)$ for the Temperature Series and the Functions $f(\alpha)$ for the Sections of Antipersistent and Persistent Dependence.

TABLE VI. HyPOTHESIS TeSting ABOUt THE MONOFRACTAL PROPERTY OF TEMPERATURE SERIES

\begin{tabular}{|l|l|l|l|l|}
\hline Series & Length & $\begin{array}{l}\text { Critical } \\
\text { value } \\
\Delta h\end{array}$ & $\begin{array}{l}\text { Observed } \\
\text { value } \Delta \hat{h}\end{array}$ & $\begin{array}{l}\text { Fractal } \\
\text { properties }\end{array}$ \\
\hline $\begin{array}{l}\text { Temperature } \\
\text { series (section 1) }\end{array}$ & \multirow{2}{*}{2000} & 0.0731 & 0.0264 & mono \\
\cline { 1 - 5 } $\begin{array}{l}\text { Temperature } \\
\text { series (section 2) }\end{array}$ & & & 0.0213 & mono \\
\hline
\end{tabular}




\section{B. Fractal Analysis of Atmospheric Layer Return Echo}

One of the methods for studying processes occurring in the atmospheric boundary layer is acoustic sounding and subsequent analysis of both echo signals and ensembles of sequentially obtained echo signal profiles - echograms. Let's conduct a fractal analysis of some of the experimental data obtained at the existing acoustic sounding station of the Kharkiv National University of Radio Electronics.

In this case, the maximum depth of the sounding atmospheric layer $Z=372 \mathrm{~m}$; the value of the resolution in depth $\Delta Z=5 \mathrm{~m}$; the carrier frequency of the sounding pulse $f=1800 \mathrm{~Hz}$, sounding pulse repetition period $T=2 \mathrm{sec}$. Fig. 15 shows graphs of signal values $X(t)$ reflected at different depth of the sounding layer $-50 \mathrm{~m}$ and $200 \mathrm{~m}$.

Signals multifractal analysis was carried out by the method of the discrete wavelet transform (MFDWT). Fig. 16 shows the dependence $\log _{2}\left(E_{j}\right)$ on the decomposition level $j$. Estimates of the Hurst exponent obtained for signals at various depths are respectively equal to $H_{50}=0.73$ and $H_{200}=0.55$.

Fig. 17 shows the values of the Hurst exponent $H$ as a function of the reflective layer depth $Z$, calculated for the numerical data of one of the echograms.

As seen from the figure, at low depth, where the proximity of buildings, structures, tall trees and other objects of the metropolis are affecting, the values of the Hurst exponent do not exceed 0.6. Then, due to the influx of heat in the lower layers of the atmosphere, the values of the Hurst exponent increase to 0.75 , which indicates the presence of long-term dependence in atmospheric processes at low depth. As the depth and distance from the megapolis increase, the value of Hurst exponent decreases and tends to 0.5 , which corresponds to weakly correlated random processes.

A multifractal analysis carried out by MFDWT confirms that with increasing depth of the reflecting atmosphere layer, the correlation structure of the processes changes, which is reflected in a decrease in the values of the generalized Hurst exponent $h(q)$. Fig. 18 shows the values of the generalized Hurst exponent for signals reflected at 50, 150, and $200 \mathrm{~m}$ depth. In this case, the values of the parameter $q$ changed in the range $1 \leq q \leq 10$.
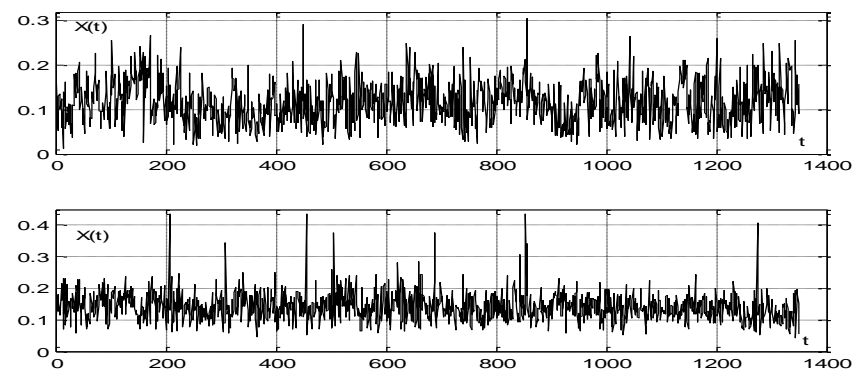

Fig. 15. Reflected Signals at $50 \mathrm{~m}$ (Top) and $200 \mathrm{~m}$ (Bottom) Depth of the Sounding Layer.

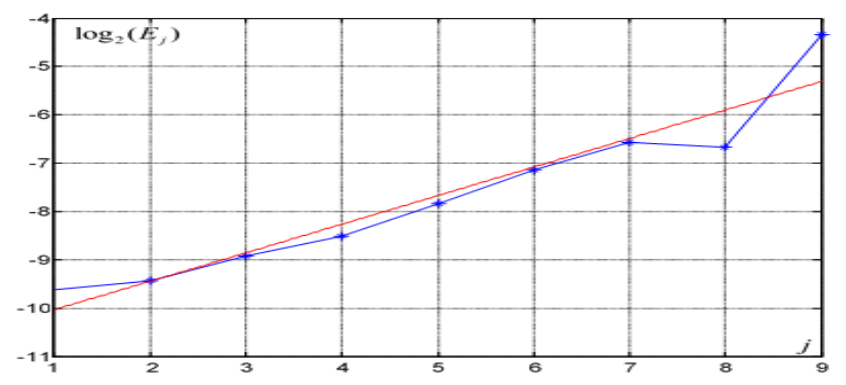

Fig. 16. Dependence $\log _{2}\left(E_{j}\right)$ on the Level $j$ for Signal Reflected at Depth $50 \mathrm{~m}$.

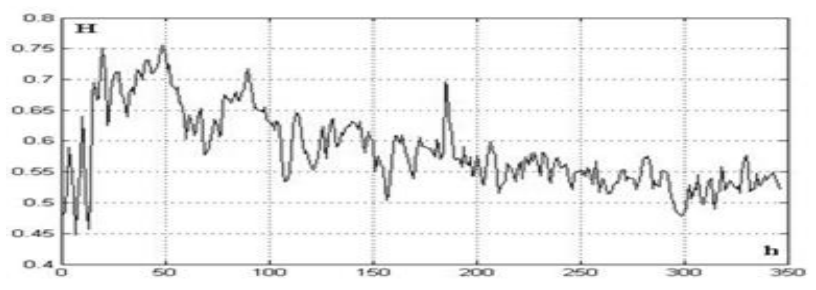

Fig. 17. Hurst Exponent $H$ as a Function of the Reflective Layer Depth.

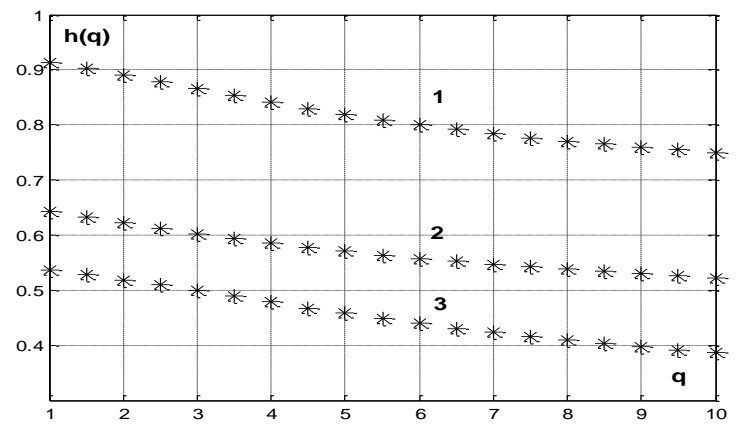

Fig. 18. Generalized Hurst Exponent for Signals Reflected at Depth $50 \mathrm{~m}$ (Line 1), $150 \mathrm{~m}$ (Line 2) and $200 \mathrm{~m}$ (Line 3).

Monofractal property testing presented in Subsection 4.3 has shown that signals reflected at different depth have weak multifractal properties. Table VII has the results of the sample generalized Hurst exponent analysis for signals of various depths. The critical value $\Delta h$ was obtained using calculated data for significance level $\alpha=0.05$ and corresponding series length.

\section{Electroencephalogram Signals Research}

Fractal geometry has been used in biology for over a quarter of a century. The use of fractal methods opens up new opportunities in studying the functional organization of living systems. Numerous experimental and clinical data lead to the conclusion that research on the fractal properties of different biological systems will lay the foundation for fractal diagnostics [29], [30].

Multifractal characteristics of electroencephalogram records of different activity.

In [31], the study of how the multifractal characteristics of the electroencephalogram records (EEG) change when a person performs a physical action, and when he imagines that he is doing it, was carried out. Experimental data were taken 
from the [32], where are the EEG of persons, who, with one signal clasped their hand into a fist, and with another signal, they only imagined that they were doing it. Fig. 19 shows typical EEG realizations in cases where the respondent squeezed his fist (left) and when the respondent imagines it (right).

Multifractal characteristics of EEG records for the abovementioned cases were studied using the method of wavelet transform modulus maxima (WTMM). Fig. 20 shows the multifractal spectrum functions $f(\alpha)$ corresponding to considered EEG records. Spectrum $f(\alpha)$ for real fist squeezed states are represented by line 1 , and line 2 corresponds to an imaginary squeezed case.

Therefore, the functions of the multifractal spectrum obtained from the EEG realization make it possible to differentiate the real and imaginary actions of the respondent.

Multifractal characteristics of EEG for different phases of wakefulness and sleep.

The EEG realizations of laboratory animals, in different phases of wakefulness and sleep, have been researched. Fig. 21 shows typical EEG for various phases: wakefulness (awake), slow-wave sleep (sws) and rapid eye movement sleep (rem).

TABLE VII. HyPOTHESIS TESTING ABOUT THE MONOFRACTAL PROPERTY OF REFLECTED SIGNALS

\begin{tabular}{|c|c|c|c|c|}
\hline Series & Length & $\begin{array}{l}\text { Critical } \\
\text { value } \Delta h\end{array}$ & $\begin{array}{l}\text { Observed } \\
\text { value } \Delta \hat{h}\end{array}$ & $\begin{array}{l}\text { Fractal } \\
\text { properties }\end{array}$ \\
\hline Depth $50 \mathrm{~m}$ & \multirow{3}{*}{1300} & \multirow{3}{*}{0.075} & 0.105 & multi \\
\hline Depth $150 \mathrm{~m}$ & & & 0.079 & multi \\
\hline Depth $200 \mathrm{~m}$ & & & 0.092 & multi \\
\hline
\end{tabular}

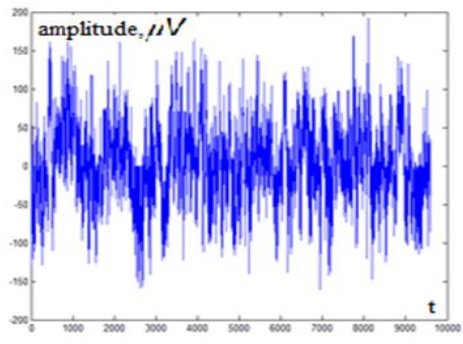

(a)

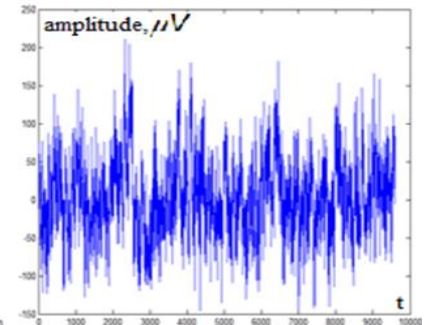

(b)
Fig. 19. EEG Records: Respondent Clenches Fist (a) and Respondent Imagines it (b).

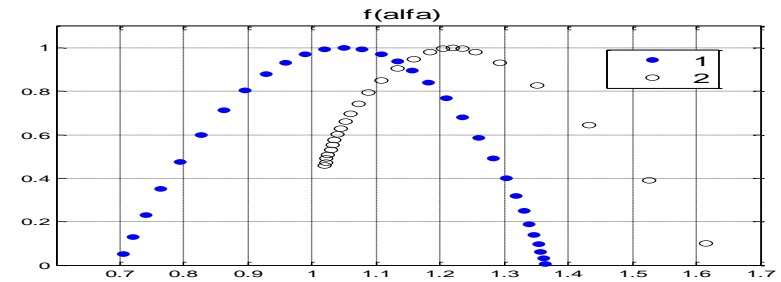

Fig. 20. Functions of $f(\alpha)$ for Really Squeezed Fist (Line 1) and Imaginary Squeezed Fist (Line 2).
Before the multifractal analysis, the research of the selfsimilar properties of the EEG realization was carried out, which identified the long-term dependence for the awake, ant persistence for the slow sleep phase and weak autocorrelation dependence for the fast sleep phase. Fig. 22 shows a fluctuation function $F(\tau)$ typical for the EEG realizations in the wakefulness.

Multifractal analysis conducted by the MFDFA method detected significant differences in fractal properties of EEG for wakefulness and sleep. Fig. 23 shows the functions of the generalized Hurst exponent $h(q)$ for the EEG realizations presented above.
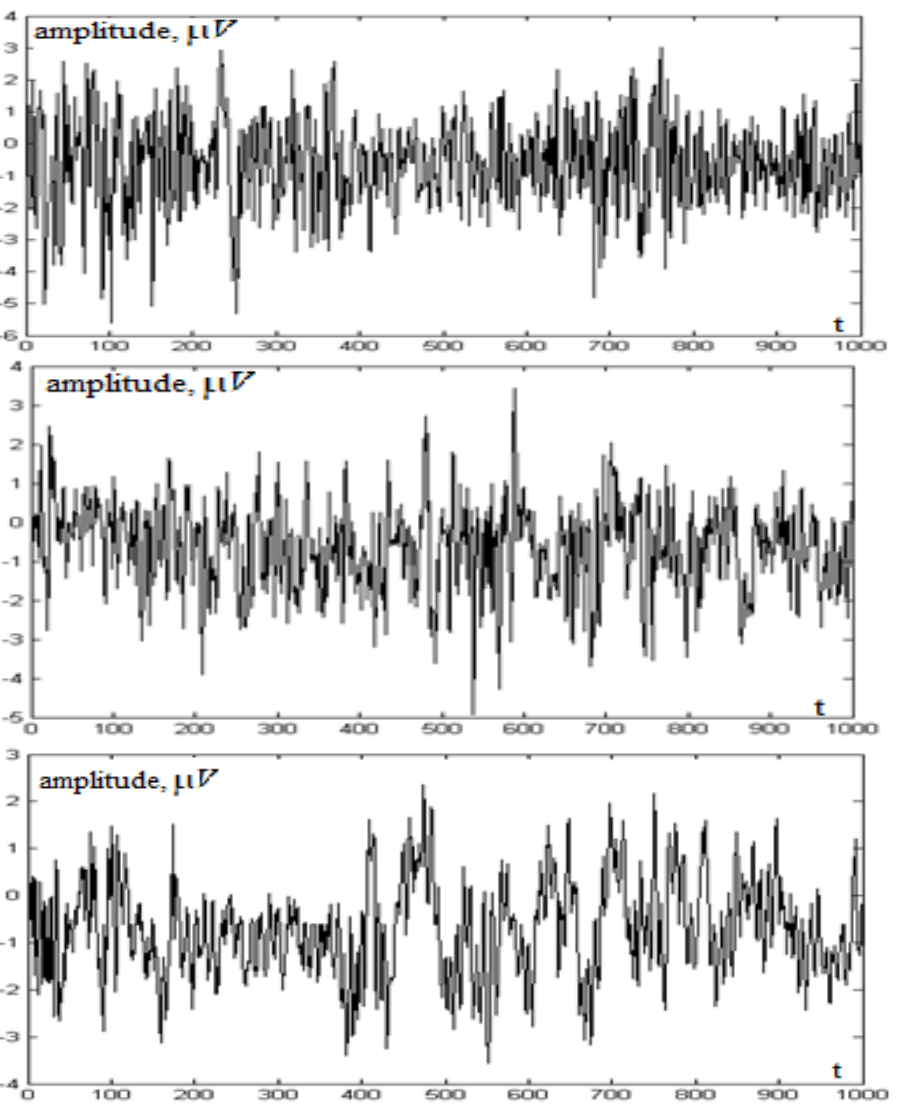

Fig. 21. EEG Realizations: awake (Top), rem (Middle) and sws (Bottom).

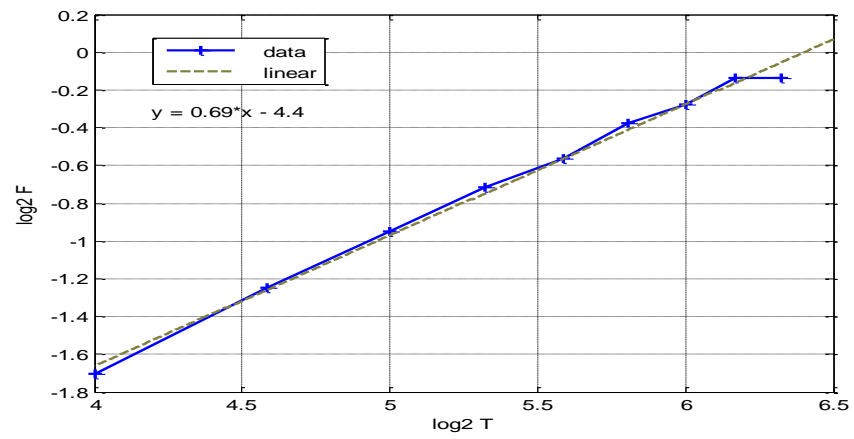

Fig. 22. Fluctuation Function $F(\tau)$ Typical for Wakefulness. 


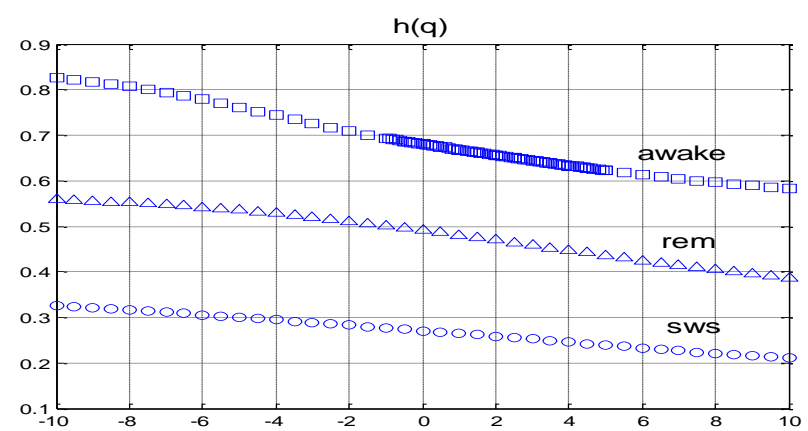

Fig. 23. Generalized Hurst Exponent for AWAKE (Squares), SWS (Circles) and REM (Triangles).

The analysis also showed that there is an undoubted longterm dependence for the EEG realizations in the awake phase: in this case, Hurst exponent $H$ is significantly exceeded 0.5 . The slow sleep phase is characterized by ant persistence, Hurst exponent takes values in the range of less than 0.5. For REM sleep, the Hurst exponent $H$ is close to 0.5 , it takes values greater or less than 0.5. In this case, the EEG realizations are characterized by very weak autocorrelation dependence.

Table VIII presents the testing results of the sample generalized Hurst exponent $\hat{h}(q)$ for the studied realizations of EEG. The critical values $\Delta h$ are based on the calculated data for significance level $\alpha=0.5$ and corresponding series length.

\section{Multifractal Analysis of Seismic Wave}

One of the known examples of time series with clearly expressed fractal properties is seismic waves. Nowadays realtime seismic monitoring is not only important but also is the most complicated task of seismological practice. There are various seismic signal detection methods based on amplitude ratio analysis, spectral characteristics, wavelet analysis, etc. Some methods based on the estimation of fractal characteristics of the seismic process such as Hurst exponent and fractal dimension [33], [34].

Numerical studies show that seismic waves have multifractal properties and different stages of their development have different functions of generalized Hurst exponent $h(q)$. Fig. 24 (top) shows a seismic time series where two windows are highlighted: before the activity burst and after. Obviously, in these windows, the function of the generalized Hurst exponent $h(q)$, shown in Fig. 24 (bottom) has significant differences.

Table IX contains the results of the analysis of generalized Hurst exponent $\hat{h}(q)$ for studied seismic wave realizations in various stages of development. Critical values $\Delta h$ are given based on calculated data for the significance level $\alpha=0.05$ and corresponding series length.

The results of the analysis allow asserting that seismic wave realizations have strong multifractal properties that allow to distinguish them from white noise which is monofractal. These properties can be used when detecting seismic signals.

\section{E. Analysis Fractal Properties of Community Activities Time} Series in Social Networks

In recent years, there have been studies of community dynamics in social groups, which show that the corresponding time series have self-similarity properties [35], [36]. The comparative fractal analysis for two groups on the social network Facebook found on keywords related to cyber threats was carried out. Two groups ThreatPost and ThreatSignal were selected to research each with 14000 and 84000 users respectively. For each of these social groups, data on the number of likes, comments, and involvement levels over the past five years has been collected.

Table $X$ presents the results of the sample generalized Hurst exponent $\hat{h}(q)$ for the studied series. The critical values $\Delta h$ are given based on calculated data for the significance level $\alpha=0.05$ and the corresponding series length.

TABLE VIII. HYPOTHESIS TESTING ABOUT MONOFRACTAL PROPERTY OF EEG

\begin{tabular}{|c|c|c|c|c|}
\hline Series & Length & $\begin{array}{l}\text { Critical } \\
\text { value } \Delta h\end{array}$ & $\begin{array}{l}\text { Observed } \\
\text { value } \Delta \hat{h}\end{array}$ & $\begin{array}{l}\text { Fractal } \\
\text { properties }\end{array}$ \\
\hline $\begin{array}{l}\text { EEG (really } \\
\text { squeezed fist) }\end{array}$ & \multirow[b]{2}{*}{2000} & \multirow[b]{2}{*}{0.0721} & 0.15 & multi \\
\hline $\begin{array}{l}\text { EEG (imaginary } \\
\text { really squeezed } \\
\text { fist) }\end{array}$ & & & 0.12 & multi \\
\hline awake EEG & \multirow{3}{*}{2500} & \multirow{3}{*}{0.0603} & 0.081 & multi \\
\hline rem EEG & & & 0.072 & multi \\
\hline sws EEG & & & 0.058 & mono \\
\hline
\end{tabular}

seismic wave amplitude
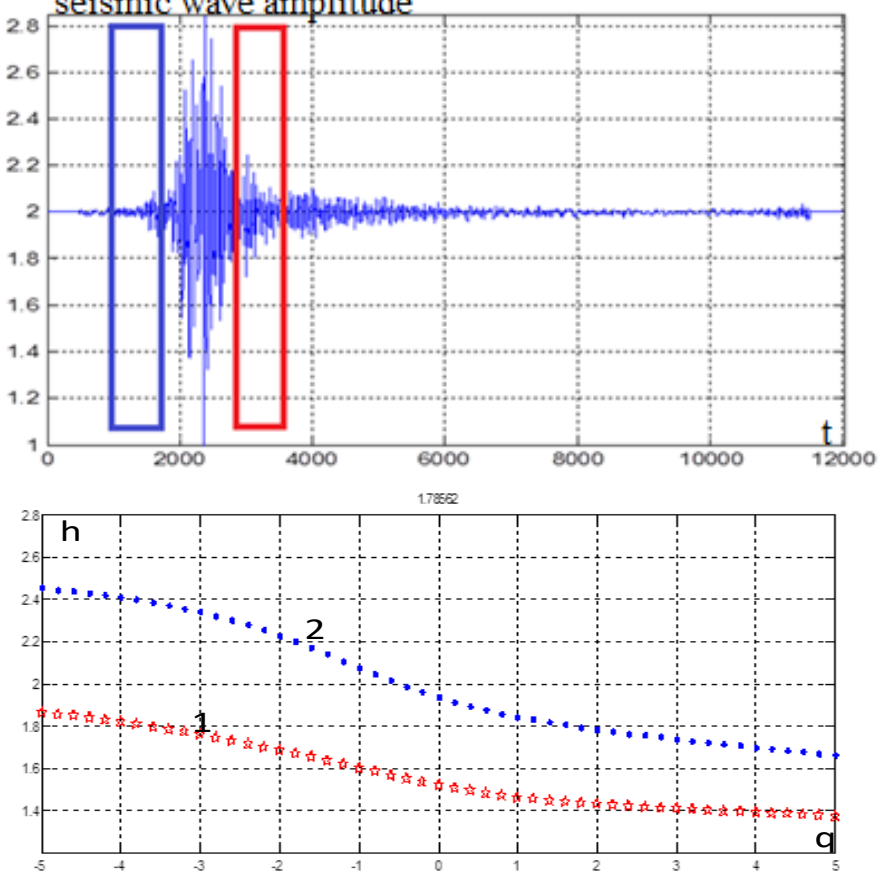

Fig. 24. Seismic Series and $h(q)$ before Burst (Line 1) and after (Line 2). 
TABLE IX. HYPOTHESIS TESTING ABOUT THE MONOFRACTAL PROPERTY OF SEISMIC WAVES

\begin{tabular}{|l|l|l|l|l|}
\hline Series & Length & $\begin{array}{l}\text { Critical } \\
\text { value } \Delta h\end{array}$ & $\begin{array}{l}\text { Observed } \\
\text { value } \Delta \hat{h}\end{array}$ & $\begin{array}{l}\text { Fractal } \\
\text { properties }\end{array}$ \\
\hline $\begin{array}{l}\text { Seismic } \\
\text { wave in the } \\
\text { initial stage }\end{array}$ & \multirow{2}{*}{1000} & 0.0861 & 0.1344 & multi \\
\cline { 1 - 4 } $\begin{array}{l}\text { Seismic } \\
\text { wave in the } \\
\text { final stage }\end{array}$ & & & 0.3212 & multi \\
\cline { 3 - 5 }
\end{tabular}

TABLE X. Hypothesis TeSting ABOUT MONOFRACTAL PROPERTy OF COMMUNITY ACTIVITIES SERIES

\begin{tabular}{|l|l|l|l|l|}
\hline \multirow{2}{*}{ Series } & Length & $\begin{array}{l}\text { Critical } \\
\text { value } \Delta h\end{array}$ & $\begin{array}{l}\text { Observed } \\
\text { value } \Delta \hat{h}\end{array}$ & $\begin{array}{l}\text { Fractal } \\
\text { properties }\end{array}$ \\
\hline ThreatPost & \multirow{2}{*}{1000} & 0.0861 & 0.9522 & multi \\
\cline { 1 - 3 } ThreatSignal & & 0.3518 & multi \\
\hline
\end{tabular}

Fig. 25 (top) shows a time series of a daily number of likes for both groups. The fractal analysis showed that these time series have strong multifractal properties. Fig. 25 (bottom) shows the values of the generalized Hurst exponent for these series. Both series have persistence $\left(H_{1}, H_{2}>0.5\right)$, but the series of likes for the group ThreatSignal has much greater fractal heterogeneity, which is expressed in a much greater range of values $\Delta h(q)$.

Research has shown that a series of likes are quite strongly correlated with the series of involvement levels, so their multifractal characteristics are very close. The series of comments have a rather close to each other multifractal structure.
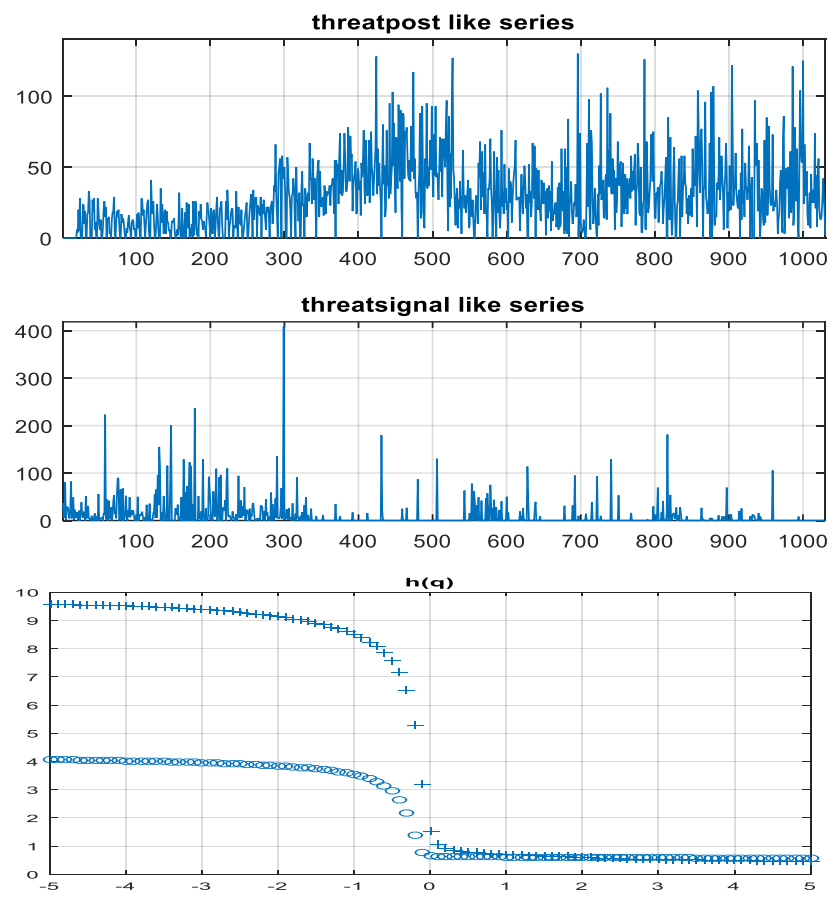

Fig. 25. Time Series of Likes for Groups and Corresponding Values $h(q)$ (+ - Group ThreatPost, o - ThreatSignal).
Thus, the research confirmed that many of the time series of social network activity indicators have fractal properties and the application of fractal analysis allows to detect differences and to reveal characteristic features of different social groups' dynamics.

\section{CONCLUSION}

The work has considered the features of the numerical implementation of multifractal analysis methods: multifractal detrended fluctuation analysis, wavelet transform modulus maxima, multifractal analysis using discrete wavelet transform. The properties of generalized Hurst exponent estimates obtained by these methods from short time series have been investigated. For this testing, each method on realizations of fractal processes of different types has been carried out. The advantages and disadvantages of each method were considered.

It is shown that the estimates of the generalized Hurst exponent, obtained from short time series, have a bias that decreases with increasing series length. It is shown that the method of multifractal detrended fluctuation analysis has significant advantages in accuracy when evaluating the characteristics of monofractal processes. A method has been developed that allows to accept or reject the hypothesis of the presence of monofractal properties in a time series. It is based on the study of sample values of the generalized Hurst exponent, obtained by the method of multifractal detrended fluctuation analysis.

Summing up the results of research, a generalized approach to the estimation of multifractal properties of time series and practical recommendations for the implementation of this approach are proposed. Examples of the practical use of the considered approach and methods for multifractal analysis of biomedical signals, natural phenomena and social networks are presented.

It should be noted certain limitations of the proposed approach. Non-stationary time series are considered in the work, but multifractal properties are assumed to be practically constant during the time realization. This requirement is consistent with the short length of the studied time series. In multifractal analysis of sufficiently long time series, it makes sense to conduct additional studies of fractal characteristics using the sliding window method.

\section{ACKNOWLEDGMENTS}

This research was supported by the Deanship of Scientific Research at Prince Sattam bin Abdulaziz University.

\section{REFERENCES}

[1] Brambila, F.: Fractal Analysis - Applications in Physics, Engineering and Technology, https://www.intechopen.com/books/fractal-analysisapplications-in-physics-engineering-and-technology, last accessed 2020/03/18.

[2] Abry, P., Goncalves P., Vehel, J. L.: Scaling, Fractals and Wavelets. John Wiley \& Sons, London (2009).

[3] Daradkeh, Y.I., Kirichenko, L., Radivilova, T.: Development of QoS Methods in the Information Networks with Fractal Traffic. International Journal of Electronics and Telecommunications 64(1), 27-32 (2018). doi: $10.24425 / 118142$. 
[4] Ageyev, D., Kirichenko, L., Radivilova, T., Tawalbeh, M., Baranovskyi, O.: Method of self-similar load balancing in network intrusion detection system. In: 2018 28th International Conference Radioelektronika (RADIOELEKTRONIKA), Prague, Czech Republic, pp. 1-4 (2018). doi: 10.1109/RADIOELEK.2018.8376406.

[5] Kirichenko, L., Bulakh, V., Radivilova, T.: Fractal time series analysis of social network activities. 2017 4th International Scientific-Practical Conference Problems of Infocommunications. Science and Technology (PIC S\&T), Kharkov, Ukraine, pp. 456-459 (2017). doi: 10.1109/INFOCOMMST.2017.8246438.

[6] Kantelhardt, J.W.: Fractal and Multifractal Time Series, 2008, https://arxiv.org/abs/0804.0747, last accessed 2020/03/18.

[7] Clegg, R. G.: A practical guide to measuring the Hurst parameter. International Journal of Simulation. Systems, Science \& Technology 7(2), 3-14 (2006).

[8] Kirichenko, L., Radivilova, T., Deineko, Z.: Comparative Analysis for Estimating of the Hurst Exponent for Stationary and Nonstationary Time Series. Information Technologies and Knowledge 5, 371-388 (2011).

[9] Xike Zhang, Gui Zhang, Luo Qiu, Bo Zhang, Yurong Sun, Zifan Gui, Qiuwen Zhang A Modified Multifractal Detrended Fluctuation Analysis (MFDFA) Approach for Multifractal Analysis of Precipitation in Dongting Lake Basin, China Water 2019, 11, 891; doi:10.3390/w11050891.

[10] Kirichenko, L., Radivilova, T., Bulakh V.: Machine Learning in Classification Time Series with Fractal Properties. Data 4(1) 5, 1-13 (2019). doi:10.3390/data4010005.

[11] L. Kirichenko, T. Radivilova and V. Bulakh, "Machine Learning in Classification Time Series with Fractal Properties." Data, vol.4(1) 5, pp.1-13, 2019. doi:10.3390/data4010005.

[12] Abry, P., Veitch, D.: Wavelet analysis of long-range dependent traffic. IEEE/ACM Transactions Information Theory 1(44), 2-15 (1998).

[13] Flandrin, P.: Wavelet Tools for Scaling Processes. Turbulence: measurements and signals. Lecture given at the Summer School, Lyon, pp.123-128 (2002).

[14] Kantelhardt, J.W., Zschiegner, S.A., Bunde, A., Havlin, S., KoscielnyBunde, E., Stanley, H.E.: Multifractal detrended fluctuation analysis of non-stationary time series. Physica A. 316, 87-114 (2002).

[15] Muzy, J.F., Bacry, E., Arneodo, A.: Wavelets and Multifractal Formalism for Singular Signals: Application to Turbulence Data. Physical review letters 67, 3515-3518 (1991).

[16] Muzy, J.F., Bacry, E., Arneodo, A.: Multifractal formalism for fractal signals: the structure-function approach versus the wavelet-transform modulus-maxima method. Physical Review E, American Physical Society (APS) 47 (2), 875-884 (1993).

[17] Veitch, D., Abry, P., Flandrin, P., Chainais, P.: Infinitely Divisible Cascade Analysis of Network Traffic Data. In: Proceedings of 2000 IEEE International Conference: Acoustics, Speech, and Signal Processing, ICASSP'00, vol.1 (2000). doi: 10.1109/ICASSP.2000.861931.

[18] Abry, P., Flandrin, P., Taqqu, M.S., Veitch, D.: Self-similarity and longrange dependence through the wavelet lens. In: Doukhan P., Oppenheim G., Taqqu M.S. (Eds.), Long Range Dependence: Theory and Applications, pp. 125-160 (2002).
[19] Oswiecimka, P., Kwapin, J., Drozdz, S.: Wavelet versus detrended fluctuation analysis of multifractal structures. Physical Review E: Statistical, Nonlinear, and Soft Matter Physics 74, 161-203 (2006).

[20] Lopez, J.L., Contreras, J.G.: Performance of multifractal detrended fluctuation analysis on short time series, https://arxiv.org/pdf/1311.2278.pdf, last accessed 2020/03/18.

[21] Kirichenko, L., Radivilova, T., Bulakh, V. Generalized approach to Hurst exponent estimating by time series. Inform. Autom. Pomiary Gospod. Ochr. Srodowiska 8, 28-31 (2018). doi:10.5604/01.3001.0010.8639.

[22] Bulakh, V., Kirichenko, L., Radivilova, T.: Time Series Classification Based on Fractal Properties. In: 2018 IEEE Second International Conference on Data Stream Mining \& Processing (DSMP), Lviv, Ukraine, pp. 198-201 (2018). doi: 10.1109/DSMP.2018.847853.

[23] Ivanisenko, I., Kirichenko, L., Radivilova, T.: Investigation of selfsimilar properties of additive data traffic. $2015 \mathrm{Xth}$ International Scientific and Technical Conference "Computer Sciences and Information Technologies" (CSIT), Lviv, pp. 169-171 (2015). doi: 10.1109/STC-CSIT.2015.7325459.

[24] Pavlov, A.N., Anishchenko, V.S.: Multifractal analysis of complex signals Physics - Uspekhi 50 (8), 819 - 834 (2007). doi: 10.1070/PU2007v050n08ABEH006116.

[25] Feder, J.: Fractals. Plenum, New York, 1988.

[26] Nakao, H.: Multi-scaling properties of truncated Levy flights. Physics Letters A 266(4-6), 282-289 (200). doi: https://doi.org/10.1016/S03759601(00)00059-1.

[27] Riedi, R.H.: Multifractal Processes. Available online: https://www.researchgate.net/publication/2839202_Multifractal_Process es, last accessed 2020/03/18.

[28] Weather archive for the cities of the CIS (19th and 20th centuries), http://thermo.karelia.ru/weather/w_history.php, last accessed 2020/03/16.

[29] Ching, E. S., Tsang, Y. K.: Multifractality and scale invariance in human heartbeat dynamics. Physical Review E 76(4), 041910 (2007).

[30] Harikrishnan, K. P., Misra, R., Ambika, G.: Can the multifractal spectrum be used as a diagnostic tool. Chaotic Modeling and Simulation 1, 51-57 (2013).

[31] Alghawli, A., Kirichenko, L.: Multifractal Properties of Bioelectric Signals under Various Physiological States. Information Content \& Processing International Journal 2(2), 138-163 (2015).

[32] PhysioNet: the research resource for complex physiologic signals, www.physionet.org, last accessed 2020/03/18.

[33] Boschetti, F., Dentith, M. D., List, R. D.: A fractal-based algorithm for detecting first arrivals on seismic traces. Geophysics 61(4), 1095-1102 (1996).

[34] Cao, M. S., Ren, Q. W., Wang, H. H., Gong, T.: A method of detecting seismic singularities using combined wavelet with fractal. Chinese journal of Geophysics 48(3), 740-749 (2005).

[35] Yang, C. C., Sageman, M.: Analysis of terrorist social networks with fractal views. Journal of Information Science 35(3), 299-320 (2009).

[36] Liu, Q., Zhao, X., Willinger, W., Wang, X., Zhao, B. Y.,Zheng, H.: Selfsimilarity in social network dynamics. ACM Transactions on Modeling and Performance Evaluation of Computing Systems (TOMPECS) 2(1), $1-26(2016)$ 